\title{
Synthesis and Antimicrobial Activity of Novel 3,7-Disubstituted 2H-1- Benzopyran-2-Ones
}

\section{Shailesh $\mathrm{K}^{1 *}$, Devdutt $\mathbf{C}^{2}$ and Devi Prasad $\mathbf{S}^{3}$}

${ }^{1}$ Department of Applied Chemistry, Babasaheb Bhimrao Ambedkar University (BBAU), Lucknow, Uttar Pradesh, India

2Department of Applied Chemistry, Amity School of Applied Sciences, Amity University Uttar Pradesh, Lucknow Campus, Lucknow, Uttar Pradesh, India ${ }^{3}$ Medicinal and Process Chemistry Division, CSIR - Central Drug Research Institute (CDRI), Lucknow, Uttar Pradesh, India

\begin{abstract}
2H-1-benzopyran-2-one (Coumarin), an important oxygen heterocyclic scaffold, widely distributed throughout the plant kingdom, displayed a wide range of potential biological activities such as anti-microbial, anti-inflammatory and antioxidant activities. In this paper, we have synthesized a novel class of 3,7-disubstituted $2 \mathrm{H}$-1-benzopyran2-one derivatives (3aa-3hb) bearing a basic ether side chain at C-7 and a substituted phenyl ring at $\mathrm{C}-3$ of the coumarin ring. These compounds have been evaluated for anti-microbial (antibacterial/antifungal) activities. Some of the compounds $\mathbf{3 a c}, \mathbf{3 a e}, \mathbf{3 b b}, \mathbf{3 b c}$ have shown significant anti-fungal activities against selective strains. Compound $3 \mathrm{ae}$ and $3 \mathrm{bc}$ with the MIC values of $1.56 \mu \mathrm{g} / \mathrm{mL}$ displayed better antifungal activity than fluconazole against Trichophyton mentagrophytes.
\end{abstract}

Keywords: Coumarins; Benzopyrans; Antimicrobial agents

\section{Introduction}

2H-1-benzopyran-2-one (Coumarin) is an important oxygen heterocyclic scaffold, widely distributed throughout the plant kingdom [1-3] and exhibited a wide range of biological activities [4] such as anticancer [5,6], anti-inflammatory [7], antioxidant [8], anti-tubercular [9], antihyperglycemic $[10,11]$, MAO-B inhibitory anticoagulation $[12,13]$ antimicrobial [14-17], (antifungal and antimicrobial) etc. It has been reported that 7 -amino substituted coumarins plays a significant role as biologically active compounds in various diseases and as substrates for P-450 isozymes [18] and 7-amino $2 \mathrm{H}$-1-benzopyran-2one derivatives isolated from Loeselia Mexicana, Petroselinum crispum, Ruta graveolens and Aesculus pavia exhibited significant antimicrobial activity [19]. A series of 7-amino-and 7-hydroxy-substituted coumarins (Figure 1, Compound I-IV), initially have been synthesized as potential zinc indicators were also possessed anti-inflammatory and antioxidant activities [20,21]. In recent years, various analogues of 3,7-disubstituted coumarins were reported as antimicrobial agents and monoamine oxidase (MAO inhibitors) [22,23]. Therefore, coumarins and their derivatives has been the subject of extensive investigations in recent years. Furthermore, it has also been realized that by incorporating substituted phenyl ring at position C-3 of the coumarin ring may increases many fold their biological activities [24]. Based on the above facts that by incorporating a basic side chain at C-7 and a substituted phenyl ring at $\mathrm{C}-3$ of coumarin ring may led to increases their biological activities. Therefore, we became interested to synthesize a compound bearing a substituted aminoethoxy chain at C-7 and a substituted phenyl ring at C-3 of coumarin ring of the designed prototype $\mathbf{V}$ (Figure 2).

Our group [25-31] has been working since several years on the design and synthesis/semisynthesis of biologically potent scaffolds for exploring their different kinds of biological activities. In the present paper, we would like to report here the synthesis and biological activities of the designed prototype $\mathbf{V}$ (Figure 2). To the best of our knowledge, 3,7-disubstituted-2 $\mathrm{H}$-1-benzopyran-2-ones bearing a basic ether side chain at position C-7 and a substituted aryl ring at position C-3 on the coumarin ring have not been studied so far till now.

\section{Chemistry}

The designed compounds 3aa-hb were synthesized from their corresponding 7-hydroxy-3-substituted 2H-1-benzopyran-2-one 2a$\mathbf{h}$, through their alkylation with different ammonium hydrochloride salts (Scheme 1). Compounds $\mathbf{2 a - h}$ were prepared by the condensation of 2,4-dihydroxybenzaldehyde with various substituted phenyl acetic acids $\mathbf{1}$ in presence of triethylamine (TEA) and acetic anhydride, which was subsequently hydrolyzed with $20 \% \mathrm{NaOH}$ afforded the 7-hydroxy derivatives $\mathbf{2 a}-\mathbf{h}$. Various kinds of the synthesized compounds $\mathbf{3 a a}-\mathbf{3 h b}$ having different $\mathrm{R}^{1}$ and $\mathrm{R}^{2}$ substituents are depicted in Tables 1 and 2.

\section{Experimental}

${ }^{1} \mathrm{H}$ NMR and ${ }^{13} \mathrm{C}$ NMR spectra were recorded on Bruker Supercon Magnet DPX-200 or DRX-300 spectrometers (operating at 200 and 300 $\mathrm{MHz}$ respectively for ${ }^{1} \mathrm{H}$; 50 and $75 \mathrm{MHz}$ respectively, for ${ }^{13} \mathrm{C}$ ) using $\mathrm{CDCl}_{3}$ and DMSO- $\mathrm{d}_{6}$ as solvent. Chemical shifts are expressed in parts per million $(\delta \mathrm{ppm}) ; J$ values are given in Hertz. Tetramethylsilane $\left(\delta 0.00 \mathrm{ppm}\right.$ ) served as an internal standard in ${ }^{1} \mathrm{H}$ NMR and $\mathrm{CDCl}_{3}$ $(\delta 77.23 \mathrm{ppm})$ in ${ }^{13} \mathrm{C}$ NMR. Splitting patterns are described as singlet (s), broad singlet (br s), broad multiplet ( $\mathrm{br} \mathrm{m}$ ), doublet (d), triplet $(\mathrm{t})$, quintet (q), septet (sep), td (doublet of triplet) and multiplet (m). Reagents and solvents used were mostly AR grade. Reaction progress was monitored by TLC aluminum sheets silica gel $60 \mathrm{~F}_{254}$. Detection of spots was done either by iodine vapors or spraying with $2 \%$ vanillin in $\mathrm{H}_{2} \mathrm{SO}_{4}$ followed by heating at $110^{\circ} \mathrm{C}$. Melting points were taken in open capillaries on an electrically heated melting point apparatus Complab and were uncorrected. IR spectra were recorded on Perkin-Elmer $\mathrm{RX}-1$ spectrophotometer using $\mathrm{KBr}$ pellets or in neat. High-resolution electron impact mass spectra (HREIMS) were obtained on JEOL MS route $600 \mathrm{H}$ instrument. Elemental analyses were performed on Vario EL-III C H N S analyzer. Column chromatography was performed over

${ }^{*}$ Corresponding author: Shailesh Kumar, Department of Applied Chemistry, Babasaheb Bhimrao Ambedkar University (BBAU), Lucknow-226 025, Uttar Pradesh, India, Tel: 8005375720; Fax: 8005375720; E-mail: drskum10@gmail.com

Received April 10, 2017; Accepted April 18, 2017; Published April 24, 2017

Citation: Shailesh K, Devdutt C, Devi Prasad S (2017) Synthesis and Antimicrobial Activity of Novel 3,7-Disubstituted 2H-1-Benzopyran-2-Ones. Med Chem (Los Angeles) 7: 857-864. doi: 10.4172/2161-0444.1000442

Copyright: @ 2017 Shailesh K, et al. This is an open-access article distributed under the terms of the Creative Commons Attribution License, which permits unrestricted use, distribution, and reproduction in any medium, provided the original author and source are credited. 
<smiles>Cc1cc(=O)oc2cc(NCCN(CCC#N)CCC#N)ccc12</smiles><smiles>Cc1cc(=O)oc2cc(NCCN(CCCl)CCCl)ccc12</smiles>

III<smiles>Cc1cc(=O)oc2cc(OCCN(CCC#N)CCC#N)ccc12</smiles>

II<smiles>COC(=O)CCN(CCNc1ccc2c(C)cc(=O)oc2c1)CCC(C)=O</smiles>

IV

Figure 1: Structures of some 7-substituted coumarins.<smiles>[R]CCOc1ccc2cc([R])c(=O)oc2c1</smiles>

V

Figure 2: Designed Prototypes (3aa-3hb).

silica gel (particle size: 60-120 Mesh) or flash silica gel (particle size: 230-400 Mesh) procured from Qualigens (India).

\section{General procedure of preparation of intermediate compounds 2H-1-benzopyran-2-ones (2a-h)}

In a $250 \mathrm{~mL}$ round bottom flask was charged successively phenyl acetic acid $\mathbf{1}$ (1.1 mmol), 2,4-dihydroxybenzaldehyde (1 mmol) and acetic anhydride $(2 \mathrm{mmol})$. After stirring for $5 \mathrm{~min}$, triethylamine $(1.4 \mathrm{mmol})$ was added dropwise over a period of $10 \mathrm{~min}$. The mixture was refluxed at $130-135^{\circ} \mathrm{C}$ for $5 \mathrm{~h}$. The reaction was monitored by TLC during the course of reaction. After completion of reaction, the reaction mixture was allowed to cool to $50-55^{\circ} \mathrm{C}$ and reaction was quenched by ice cooled water $(200 \mathrm{~mL})$ with continuous stirring for $15 \mathrm{~min}$. The solid obtained was filtered and washed with ice cooled water $(50 \mathrm{~mL} \times 3)$. The wet solid product was placed in $250 \mathrm{~mL}$ flask and $15 \mathrm{ml}$ of $20 \% \mathrm{NaOH}$ solution was added. The mixture is stirred for $1 \mathrm{~h}$ at $50-55^{\circ} \mathrm{C}$ and then cooled to $15^{\circ} \mathrm{C}$, and acidified with $5 \mathrm{~N} \mathrm{HCl}$ till acidic to litmus. The precipitated product was filtered, washed with ice cold water $(50 \mathrm{~mL} \times 3)$ and sucked dry. The product was further dried at $65^{\circ} \mathrm{C}$ under vacuum to afford the 3-substituted-7- hydroxyl- $2 \mathrm{H}-1$ benzopyran-2-one in $70-80 \%$ yield.

7-Hydroxy-3-phenyl- 2H-1-benzopyran-2-one (2a): Yield 68\%; mp $195-197^{\circ} \mathrm{C}$; ${ }^{1} \mathrm{H}$ NMR $\left(300 \mathrm{MHz}, \mathrm{CDCl}_{3}+\mathrm{DMSO}-d_{6}\right) \delta 6.84(\mathrm{dd}$, $1 \mathrm{H}, J=8.5,2.3 \mathrm{~Hz}), 6.87(\mathrm{~d}, 1 \mathrm{H}, J=2.0 \mathrm{~Hz}), 7.31-7.42(\mathrm{~m}, 5 \mathrm{H}), 7.46-7.49$ $(\mathrm{m}, 1 \mathrm{H}), 7.67(\mathrm{~s}, 1 \mathrm{H}) ;{ }^{13} \mathrm{C} \mathrm{NMR}\left(75 \mathrm{MHz}, \mathrm{CDCl}_{3}+\mathrm{DMSO}-d_{6}\right) \delta 102.53$,
$111.35,113.51,121.87,126.56,129.06,129.41,131.30,133.42,134.09$, $142.99,155.51,161.52$; ES-MS $(m / z) 239[\mathrm{M}+\mathrm{H}]^{+}$.

7-Hydroxy-3-(2-methoxy-phenyl)- 2H-1-benzopyran-2-one (2c): Yield 70\%; mp 180- $182^{\circ} \mathrm{C} ;{ }^{1} \mathrm{H}$ NMR $\left(300 \mathrm{MHz}, \mathrm{CDCl}_{3}+\mathrm{DMSO}-d_{6}\right)$ $\delta 3.82(\mathrm{~s}, 3 \mathrm{H}), 6.80-6.84(\mathrm{~m}, 2 \mathrm{H}), 6.97-7.03(\mathrm{~m}, 2 \mathrm{H}), 7.33-7.38(\mathrm{~m}, 3 \mathrm{H})$, $7.68(\mathrm{~s}, 1 \mathrm{H}), 9.95(\mathrm{~s}, 1 \mathrm{H}, \mathrm{OH}) ;{ }^{13} \mathrm{C}$ NMR $\left(75 \mathrm{MHz}, \mathrm{CDCl}_{3}+\mathrm{DMSO}-d_{6}\right)$ $\delta 55.58,102.57,111.13,112.03,113.30,120.36,121.47,124.40,128.82$, 129.61, 130.73, 140.72, 142.27, 155.32, 157.09, 160.98; ES-MS $(\mathrm{m} / z) 269$ $[\mathrm{M}+\mathrm{H}]^{+}$.

3-(3,4-Dimethoxy-phenyl)-7-hydroxy- 2H-1-benzopyran-2-one (2d): Yield 67\%; $\mathrm{mp} 193-195^{\circ} \mathrm{C} ;{ }^{1} \mathrm{H}$ NMR $\left(300 \mathrm{MHz}, \mathrm{CDCl}_{3}+\mathrm{DMSO}-d_{6}\right)$ $\delta 3.92(\mathrm{~s}, 3 \mathrm{H}), 3.94(\mathrm{~s}, 3 \mathrm{H}), 6.81-6.86(\mathrm{~m}, 2 \mathrm{H}), 6.93(\mathrm{~d}, 1 \mathrm{H}, J=8.3 \mathrm{~Hz})$, 7.24-7.29 (m, 3H), $7.36(\mathrm{~d}, 1 \mathrm{H}, J=8.4 \mathrm{~Hz}), 7.71(\mathrm{~s}, 1 \mathrm{H}), 9.57(\mathrm{~s}, 1 \mathrm{H}, \mathrm{OH})$; ES-MS $(m / z) 299[\mathrm{M}+\mathrm{H}]^{+}$.

7-Hydroxy-3-naphthalen-1-yl-2H-1-benzopyran-2-one (2e): Yield 70\%; mp $183-185^{\circ} \mathrm{C} ;{ }^{1} \mathrm{H}$ NMR $\left(300 \mathrm{MHz}, \mathrm{CDCl}_{3}\right) \delta 6.85(\mathrm{dd}, 1 \mathrm{H}$, $J=8.5,2.3 \mathrm{~Hz}), 6.94(\mathrm{~d}, 1 \mathrm{H}, J=2.0 \mathrm{~Hz}), 7.36(\mathrm{~d}, 1 \mathrm{H}, J=8.5 \mathrm{~Hz}), 7.44-7.55$ (m, 4H), $7.74(\mathrm{~s}, 1 \mathrm{H}), 7.78-7.81(\mathrm{~m}, 1 \mathrm{H}), 7.88-7.91(\mathrm{~m}, 2 \mathrm{H}), 9.86(\mathrm{~s}$, $1 \mathrm{H}, \mathrm{OH}) ;{ }^{13} \mathrm{C}$ NMR $\left(50 \mathrm{MHz}, \mathrm{CDCl}_{3}\right) \delta 103.01,112.14,113.85,123.45$, $125.39,126.04,126.38,127.78,128.53,129.02,129.16,131.82,133.36$, 133.73, 143.54, 155.90, 161.55; ES-MS $(\mathrm{m} / z) 289[\mathrm{M}+\mathrm{H}]^{+}$.

3-(4-Fluoro-phenyl)-7-hydroxy-2H-1-benzopyran-2-one (2g): Yield 64\%; mp 243-245 ${ }^{\circ} \mathrm{C}$; ${ }^{1} \mathrm{H}$ NMR $\left(300 \mathrm{MHz}, \mathrm{CDCl}_{3}+\mathrm{DMSO}-d_{6}\right.$ ) $\delta$ 6.81-6.86 (m, 2H), 7.08-7.14 (m, 2H), 7.33-7.38 (m, 1H), 7.64$7.69(\mathrm{~m}, 2 \mathrm{H}), 7.73(\mathrm{~s}, 1 \mathrm{H}), 9.86(\mathrm{~s}, 1 \mathrm{H}, \mathrm{OH}) ;{ }^{13} \mathrm{C} \mathrm{NMR}(75 \mathrm{MHz}$, $\left.\mathrm{CDCl}_{3}+\mathrm{DMSO}-d_{6}\right) \delta 101.77,111.50,113.12,114.36,114.64,121.45$, $128.65,129.50,129.61,130.76,140.03,154.62,160.88$; ES-MS $(\mathrm{m} / z) 257$ $[\mathrm{M}+\mathrm{H}]^{+}$.

\section{General procedure of preparation of compound (3aa-hb)}

To a solution of $2 \mathrm{H}$-1-benzopyran-2-one $\mathbf{2 a - h}(1 \mathrm{mmol})$ in $5 \mathrm{~mL}$ of dry acetone was added anhydrous potassium carbonate $(3 \mathrm{mmol})$ and corresponding ammonium hydrochloride salt $(1.2 \mathrm{mmol})$. The mixture was then refluxed for $3 \mathrm{~h}$. The reaction mixture was cooled at room temperature and filtered through sintered funnel. The residue was washed with acetone $(10 \mathrm{~mL} \times 3)$. The filtrate was then concentrated to obtain a viscous liquid which was purified by silica gel column chromatography using $2-5 \%$ methanol-chloroform mixture as eluent. 


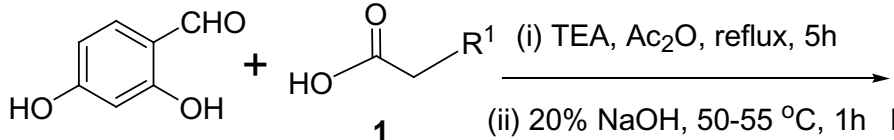

(ii) $20 \% \mathrm{NaOH}, 50-55^{\circ} \mathrm{C}, 1 \mathrm{~h}$ HO

(iii) amine hydrochloride (5) $\mathrm{K}_{2} \mathrm{CO}_{3}$, Acetone, $3 \mathrm{~h}$.

\section{2a: phenyl}

2b: 2-chlorophenyl

2c: 2-methoxyphenyl

2d: 3,4-dimethoxyphenyl

2e: 1-naphthyl

2f: 3,4-dichlorophenyl

2g: 4-fluorophenyl

2h: 4-chlorophenyl<smiles>[R]CCOc1ccc2cc([R])c(=O)oc2c1</smiles>

3aa-3hb

Scheme 1: Synthesized compounds 3aa-hb.

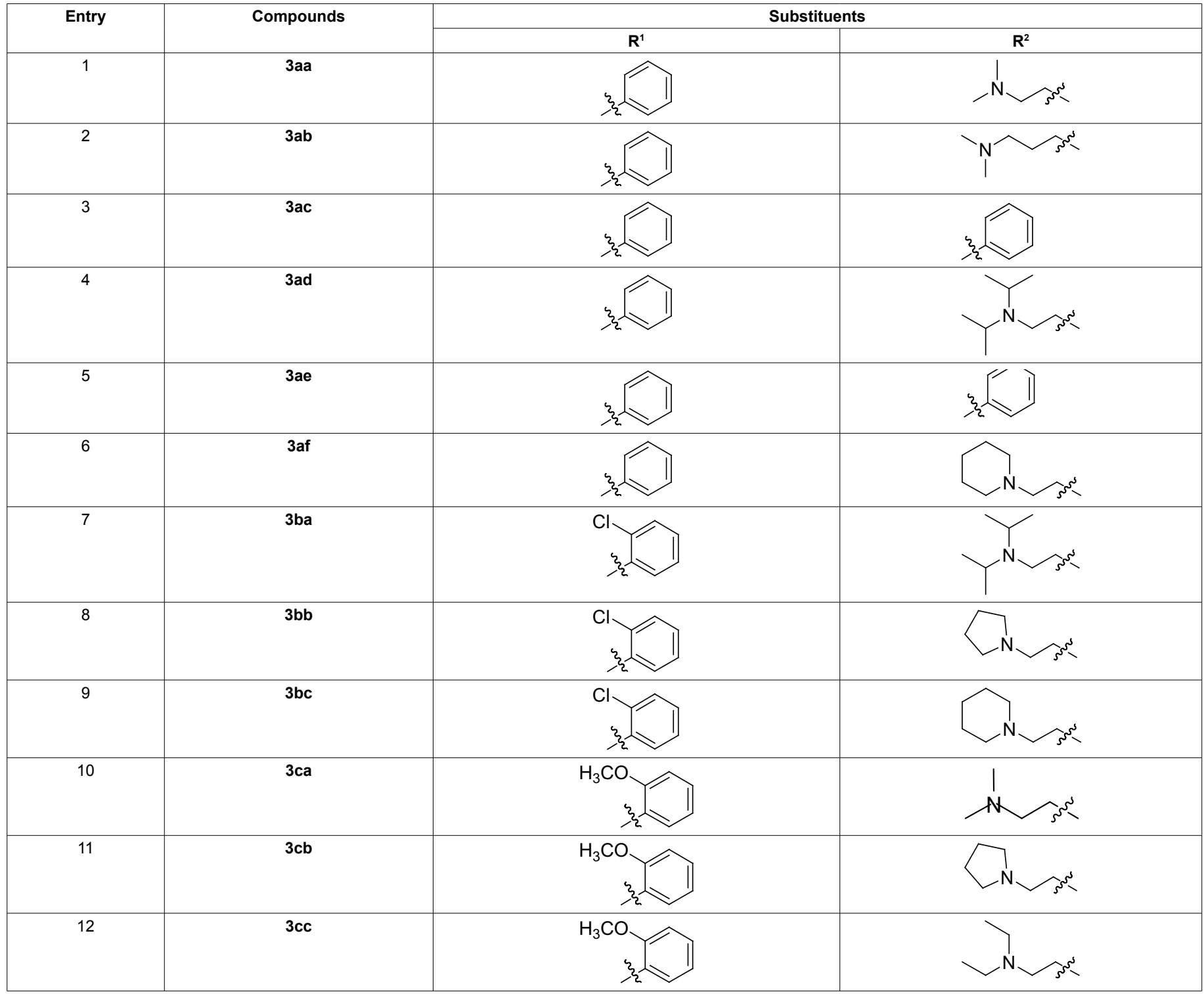




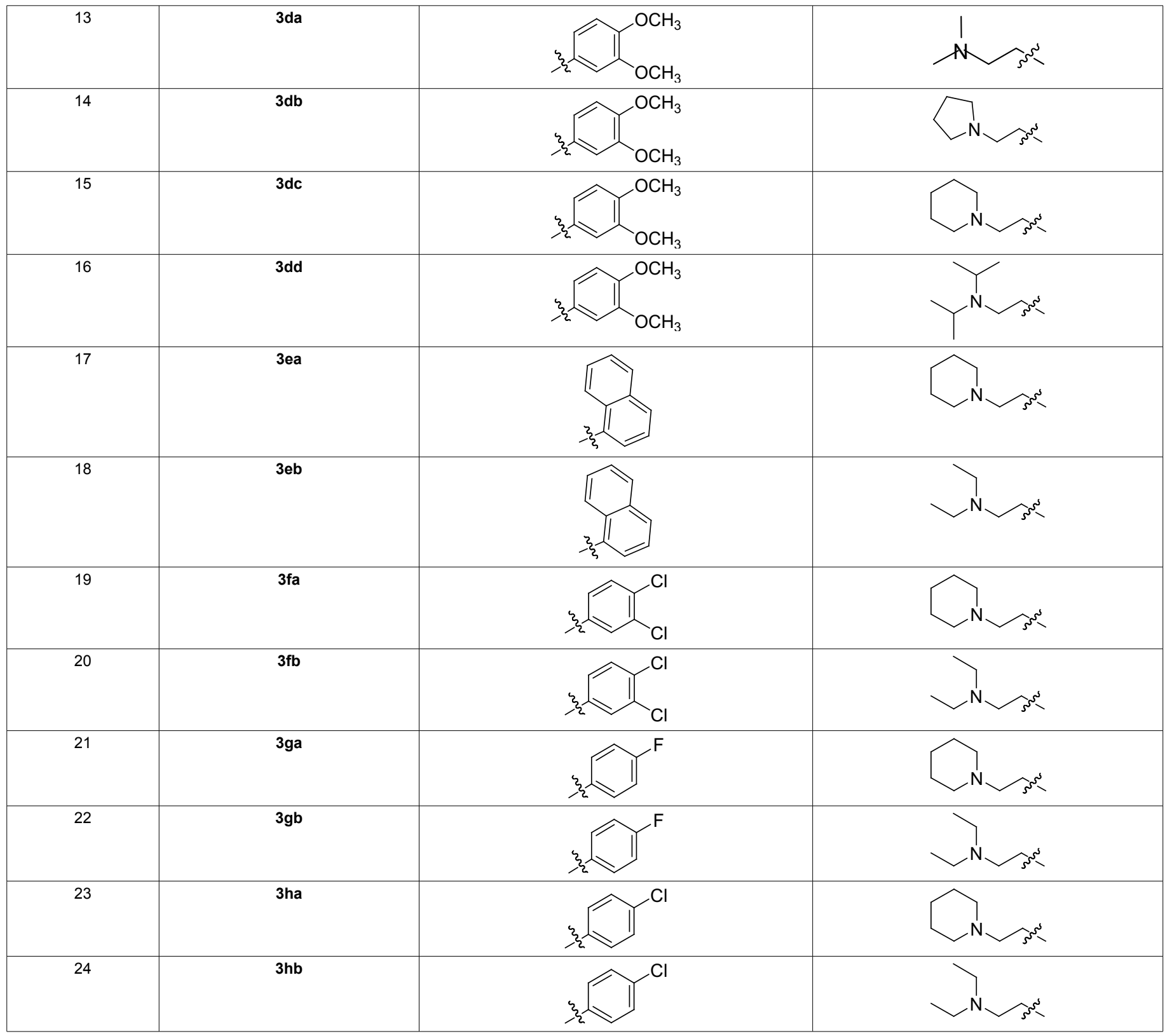

Table 1: Synthesized compounds 3aa-3hb.

\begin{tabular}{|c|c|c|c|c|c|c|c|c|c|c|c|}
\hline \multirow[t]{3}{*}{ Entry } & \multirow[t]{3}{*}{ Compound } & \multicolumn{10}{|c|}{ MIC $(\mu \mathrm{g} / \mathrm{mL})$} \\
\hline & & \multicolumn{4}{|c|}{ Bacteria } & \multicolumn{6}{|c|}{ Fungi } \\
\hline & & 1 & 2 & 3 & 4 & 5 & 6 & 7 & 8 & 9 & 10 \\
\hline 1 & 3aa & - & - & - & - & 50 & 25 & 25 & 12.5 & 50 & 50 \\
\hline 2 & $3 a b$ & - & - & - & - & 12.5 & 12.5 & 25 & 12.5 & 50 & 50 \\
\hline 3 & 3ac & - & - & - & - & 1.56 & 1.56 & 50 & 12.5 & 50 & 50 \\
\hline 4 & 3ad & - & - & - & - & - & - & 50 & 25 & - & - \\
\hline 5 & $3 a e$ & - & - & 25 & 50 & 12.5 & 12.5 & 25 & 1.56 & 12.5 & 25 \\
\hline 6 & 3af & 50 & 50 & - & - & 6.25 & 12.5 & 6.25 & 6.25 & 25 & 25 \\
\hline 7 & $3 \mathrm{ba}$ & - & - & - & - & - & - & 12.5 & 25 & - & - \\
\hline 8 & $3 b b$ & - & 50 & - & - & 50 & 50 & 25 & 3.12 & 20 & 50 \\
\hline 9 & $3 b c$ & - & - & - & - & 50 & 50 & 12.5 & 1.56 & 25 & - \\
\hline 10 & $3 \mathrm{ca}$ & - & - & - & - & - & - & - & 50 & - & - \\
\hline 11 & $3 \mathrm{cb}$ & - & 50 & - & - & - & - & - & 25 & - & - \\
\hline 12 & $3 c c$ & - & - & - & - & - & - & - & 50 & - & - \\
\hline
\end{tabular}


Citation: Shailesh K, Devdutt C, Devi Prasad S (2017) Synthesis and Antimicrobial Activity of Novel 3,7-Disubstituted 2H-1-Benzopyran-2-Ones. Med Chem (Los Angeles) 7: 857-864. doi: 10.4172/2161-0444.1000442

\begin{tabular}{|c|c|c|c|c|c|c|c|c|c|c|c|}
\hline 13 & $\mathbf{3 d a}$ & 50 & 50 & - & 50 & 50 & - & 50 & 50 & - & 50 \\
\hline 14 & $\mathbf{3 d b}$ & - & - & - & - & 50 & 50 & - & 25 & - & - \\
\hline 15 & $\mathbf{3 d c}$ & - & - & - & - & - & - & - & 50 & - & - \\
\hline 16 & $\mathbf{3 d d}$ & - & - & - & - & - & 50 & - & 50 & - & - \\
\hline 17 & $\mathbf{3 e a}$ & 50 & 50 & 50 & 50 & 6.25 & 25 & 12.5 & 12.5 & 25 & 12.5 \\
\hline 18 & $\mathbf{3 e b}$ & - & 50 & - & - & 12.5 & - & 6.25 & 12.5 & 25 & 25 \\
\hline 19 & $\mathbf{3 f a}$ & - & - & - & 50 & 12.5 & 25 & 12.5 & 6.25 & 12.5 & 6.25 \\
\hline 20 & $\mathbf{3 f b}$ & 50 & 50 & - & 50 & 12.5 & 25 & 12.5 & 6.25 & 25 & 25 \\
\hline 21 & $\mathbf{3 g a}$ & 50 & 50 & - & - & 50 & 50 & 50 & 12.5 & - & 12.5 \\
\hline 22 & $\mathbf{3 g b}$ & - & - & - & - & $\mathbf{3 . 1 2}$ & - & 12.5 & 12.5 & 50 & 6.25 \\
\hline 23 & $\mathbf{3 h a}$ & 50 & 50 & - & - & $\mathbf{3 . 1 2}$ & - & 12.5 & 12.5 & - & 12.5 \\
\hline 24 & $\mathbf{3 h b}$ & - & 50 & - & - & $\mathbf{3 . 1 2}$ & - & 6.25 & 6.25 & 25 & 50 \\
\hline flu* & ND & ND & ND & ND & ND & 0.5 & 1.0 & 1.0 & 2.0 & 2.0 & 1.0 \\
\hline
\end{tabular}

1. E. coli (ATCC 9637); 2. Pseudomonas aeruginosa (ATCC BAA-427); 3. Staphyloccus aerus (ATCC 25923); 4. Klebsiella pneumoniae (ATCC 27736); 5. Candida albicans; 6. Cryptococcus neoformans; 7. Sporothrix schenckii; 8. Trichophyton mentagrophytes; 9 Aspergillus fumigatus; 10. Candida parapsilosis (ATCC- 22019); Flu*=Fluconazole; '-' indicates MIC values above $50 \mu \mathrm{g} / \mathrm{mL}$; ND: Not done.

Table 2: In-vitro antimicrobial activities of 3-aryl-7-alkylaminoethoxy $2 \mathrm{H}$-1-benzopyran-2-one (3aa-hb).

7-(2-Dimethylamino ethoxy)-3-phenyl 2H-1-benzopyran-2-one (3aa): Yield 81\%; mp 96-98 ${ }^{\circ} \mathrm{C} ;{ }^{1} \mathrm{H}$ NMR $\left(400 \mathrm{MHz}, \mathrm{CDCl}_{3}\right) \delta 2.36(\mathrm{~s}$, $6 \mathrm{H}), 2.78(\mathrm{t}, 2 \mathrm{H}, J=5.6 \mathrm{~Hz}), 4.14(\mathrm{t}, 2 \mathrm{H}, J=5.6 \mathrm{~Hz}), 6.87-6.93(\mathrm{~m}, 2 \mathrm{H})$ 7.38-7.47 (m, 4H), 7.67-7.71 (m, 2H), $7.76(\mathrm{~s}, 1 \mathrm{H}) ;{ }^{13} \mathrm{C} \mathrm{NMR}(75 \mathrm{MHz}$ $\left.\mathrm{CDCl}_{3}\right) \delta 46.11\left(\mathrm{NCH}_{3} \times 2\right), 58.24\left(\mathrm{NCH}_{2}\right), 66.89\left(\mathrm{OCH}_{2}\right), 101.29(\mathrm{CH})$, $113.48(\mathrm{CH}), 113.66(\mathrm{C}), 128.64(\mathrm{CH} \times 3), 129.03(\mathrm{CH}), 135.28(\mathrm{C})$, $140.19(\mathrm{CH}), 144.51(\mathrm{C}), 155.50(\mathrm{C}), 161.09(\mathrm{C}), 162.09(\mathrm{CO}) ; v_{\max }(\mathrm{KBr}$, $\left.\mathrm{cm}^{-1}\right)$ 1670, 1598, 1527, 1216 761; ES-MS $(\mathrm{m} / z) 310[\mathrm{M}+\mathrm{H}]^{+}$. Found C 73.87\%, H 6.21\%, N 4.55\%; $\mathrm{C}_{19} \mathrm{H}_{19} \mathrm{NO}_{3}$ requires C 73.80\%, $\mathrm{H} 6.19 \%, \mathrm{~N}$ $4.53 \%$.

7-(3-Dimethylaminopropoxy)-3-phenyl-2H-1-benzopyran-2one (3ab): Yield $83 \%$; $\mathrm{mp} 94-96^{\circ} \mathrm{C} ;{ }^{1} \mathrm{H}$ NMR $\left(300 \mathrm{MHz} \mathrm{CDCl}_{3}\right) \delta 2.00$ (quin, $2 \mathrm{H}, J=7.1 \mathrm{~Hz}), 2.27(\mathrm{~s}, 6 \mathrm{H}), 2.48(\mathrm{t}, 2 \mathrm{H}, J=7.1 \mathrm{~Hz}), 4.09(\mathrm{t}, 2 \mathrm{H}$, $J=6.3 \mathrm{~Hz}), 6.86-6.89(\mathrm{~m}, 2 \mathrm{H}), 7.35-7.47(\mathrm{~m}, 4 \mathrm{H}), 7.68-7.71(\mathrm{~m}, 2 \mathrm{H})$, $7.76(\mathrm{~s}, 1 \mathrm{H}) ;{ }^{13} \mathrm{C} \mathrm{NMR}\left(75 \mathrm{MHz}, \mathrm{CDCl}_{3}\right) \delta 27.47\left(\mathrm{CH}_{2} \mathrm{CH}_{2} \mathrm{CH}_{2}\right), 45.72$ $\left(\mathrm{NCH}_{3} \times 2\right), 56.34\left(\mathrm{NCH}_{2}\right), 67.02\left(\mathrm{OCH}_{2}\right), 101.23(\mathrm{CH}), 113.30(\mathrm{CH})$, $113.48(\mathrm{C}), 124.92(\mathrm{C}), 128.61(\mathrm{CH}), 128.64(\mathrm{CH}), 129.02(\mathrm{CH}), 135.27$ (C), $140.31(\mathrm{CH}), 155.50(\mathrm{C}), 161.20(\mathrm{C}), 162.28(\mathrm{CO}) ; v_{\max }(\mathrm{KBr}, \mathrm{cm}$ 1) $1711,1611,1217,1008$ 780; ES-MS $(m / z) 324[\mathrm{M}+\mathrm{H}]^{+}$. Found C $74.37 \%, \mathrm{H} 6.49 \%, \mathrm{~N} 4.32 \% ; \mathrm{C}_{20} \mathrm{H}_{21} \mathrm{NO}_{3}$ requires $\mathrm{C} 74.28 \%, \mathrm{H} 6.55 \%$, $\mathrm{N} 4.33 \%$.

7-(2-Diethylaminoethoxy)-3-phenyl -2H-1-benzopyran-2-one (3ac): Yield 82\%; mp 66-68 ${ }^{\circ} \mathrm{C} ;{ }^{1} \mathrm{H}$ NMR $\left(300 \mathrm{MHz}, \mathrm{CDCl}_{3}\right) \delta 1.08(\mathrm{t}$, $6 \mathrm{H}, J=7.1 \mathrm{~Hz}), 2.65(\mathrm{q}, 4 \mathrm{H}, J=7.1 \mathrm{~Hz}), 2.90(\mathrm{t}, 2 \mathrm{H}, J=6.1 \mathrm{~Hz}), 4.11(\mathrm{t}$, $2 \mathrm{H}, J=6.1 \mathrm{~Hz}), 6.86-6.89(\mathrm{~m}, 2 \mathrm{H}), 7.34-7.46(\mathrm{~m}, 4 \mathrm{H})$, 7.67-7.70 $(\mathrm{m}, 2 \mathrm{H})$, $7.75(\mathrm{~s}, 1 \mathrm{H}) ;{ }^{13} \mathrm{C}$ NMR $\left(50 \mathrm{MHz}, \mathrm{CDCl}_{3}\right) \delta 12.07\left(\mathrm{NCH}_{2} \mathrm{CH}_{3} \times 2\right), 48.12$ $\left(\mathrm{NCH}_{2} \mathrm{CH}_{3} \times 2\right), 51.74\left(\mathrm{NCH}_{2}\right), 67.60\left(\mathrm{OCH}_{2}\right), 101.26(\mathrm{CH}), 113.39$ $(\mathrm{CH}), 113.54(\mathrm{C}), 124.97(\mathrm{C}), 128.60(\mathrm{CH}), 128.99(\mathrm{CH}), 135.26(\mathrm{C})$, $140.22(\mathrm{CH}), 155.47(\mathrm{C}), 161.10(\mathrm{C}), 162.15(\mathrm{CO}) ; v_{\max }\left(\mathrm{KBr}, \mathrm{cm}^{-1}\right) 1721$, $1613,1215,768$; ES-MS $(m / z) 338[\mathrm{M}+\mathrm{H}]^{+}$. Found C 74.80\%, H 6.84\%, $\mathrm{N} 4.18 \% ; \mathrm{C}_{21} \mathrm{H}_{23} \mathrm{NO}_{3}$ requires C 74.75\%, H 6.87\%, N 4.15\%.

7-(2-Diisopropylaminoethoxy)-3-phenyl- 2H-1-benzopyran-2one (3ad): Yield $79 \% ; \mathrm{mp} 87-89^{\circ} \mathrm{C}$; ${ }^{1} \mathrm{H}$ NMR $\left(300 \mathrm{MHz}, \mathrm{CDCl}_{3}\right) \delta 1.06$ $(\mathrm{d}, 12 \mathrm{H}, J=6.5 \mathrm{~Hz}), 2.86(\mathrm{t}, 2 \mathrm{H}, J=7.2 \mathrm{~Hz}), 3.06$ (sep, $2 \mathrm{H}, J=6.5 \mathrm{~Hz}), 3.97$ $(\mathrm{t}, 2 \mathrm{H}, J=7.2 \mathrm{~Hz}), 6.85-6.88(\mathrm{~m}, 2 \mathrm{H}), 7.35-7.47(\mathrm{~m}, 4 \mathrm{H}), 7.67-7.70(\mathrm{~m}$, $2 \mathrm{H}), 7.76(\mathrm{~s}, 1 \mathrm{H}) ;{ }^{13} \mathrm{C} \mathrm{NMR}\left(75 \mathrm{MHz}, \mathrm{CDCl}_{3}\right) \delta 21.10\left(\mathrm{NCHCH}_{3} \times 4\right)$, $44.36\left(\mathrm{NCH}_{2} \times 2\right), 49.88(\mathrm{CH} \times 2), 70.11\left(\mathrm{OCH}_{2}\right), 101.35(\mathrm{CH}), 113.27$ $(\mathrm{CH}), 113.44(\mathrm{C}), 124.86(\mathrm{C}), 128.60(\mathrm{CH}), 129.01(\mathrm{CH}), 135.31(\mathrm{C})$, $140.26(\mathrm{CH}), 155.53(\mathrm{C}), 161.15(\mathrm{C}), 162.31(\mathrm{CO}) ; v_{\text {max }}\left(\mathrm{KBr}, \mathrm{cm}^{-1}\right) 1715$, $1622,1283,1144,784$; ES-MS $(\mathrm{m} / z) 366[\mathrm{M}+\mathrm{H}]^{+}$. Found C 75.68\%, H 7.35\%, N 3.87\%; $\mathrm{C}_{23} \mathrm{H}_{27} \mathrm{NO}_{3}$ requires C 75.59\%, H 7.45\%, N 3.83\%.
3-Phenyl-7-(2-pyrrolidin-1-yl-ethoxy)- 2H-1-benzopyran-2one (3ae): Yield 85\%; mp 130-132 ${ }^{\circ} \mathrm{C} ;{ }^{1} \mathrm{H}$ NMR $\left(200 \mathrm{MHz}, \mathrm{CDCl}_{3}\right) \delta$ 1.76-1.92 (m, 4H), 2.61-2.70 (m, 4H), $2.95(\mathrm{t}, 2 \mathrm{H}, J=5.8 \mathrm{~Hz}), 4.18(\mathrm{t}$, $2 \mathrm{H}, J=5.8 \mathrm{~Hz}), 6.86-6.92(\mathrm{~m}, 2 \mathrm{H}), 7.37-7.48(\mathrm{~m}, 4 \mathrm{H}), 7.66-7.71(\mathrm{~m}$, $2 \mathrm{H}), 7.76(\mathrm{~s}, 1 \mathrm{H}) ;{ }^{13} \mathrm{C} \mathrm{NMR}\left(50 \mathrm{MHz}, \mathrm{CDCl}_{3}\right) \delta 23.72\left(\mathrm{NCH}_{2} \mathrm{CH}_{2} \times 2\right)$, $54.98\left(\mathrm{NCH}_{2} \times 3\right), 67.95\left(\mathrm{OCH}_{2}\right), 101.29(\mathrm{CH}), 113.43(\mathrm{CH}), 113.59(\mathrm{C})$, $125.03(\mathrm{C}), 128.61(\mathrm{CH}), 129.02(\mathrm{CH}), 135.25(\mathrm{C}), 140.22(\mathrm{CH}), 155.46$ (C), $161.12(\mathrm{C}), 162.08(\mathrm{CO}) ; v_{\max }\left(\mathrm{KBr}, \mathrm{cm}^{-1}\right) 1719,1598,1267,780$; ESMS $(m / z) 336[\mathrm{M}+\mathrm{H}]^{+}$. Found C 75.29\%, H 6.29\%, N 4.2\%; $\mathrm{C}_{21} \mathrm{H}_{21} \mathrm{NO}_{3}$ requires $\mathrm{C} 75.20 \%, \mathrm{H} 6.31 \%, \mathrm{~N} 4.18 \%$.

3-Phenyl-7-(2-piperidin-1-yl-ethoxy)-2H-1-benzopyran-2one (3af): Yield $86 \%$; mp $101-103^{\circ} \mathrm{C}$; ${ }^{1} \mathrm{H}$ NMR $\left(300 \mathrm{MHz}, \mathrm{CDCl}_{3}\right.$ ) $\delta 1.46-1.49(\mathrm{~m}, 2 \mathrm{H}), 1.59-1.66(\mathrm{~m}, 4 \mathrm{H}), 2.53(\mathrm{br} \mathrm{m} 4 \mathrm{H}), 2.81(\mathrm{t}, 2 \mathrm{H}$, $J=5.9 \mathrm{~Hz}), 4.18(\mathrm{t}, 2 \mathrm{H}, J=5.9 \mathrm{~Hz}), 6.87-6.90(\mathrm{~m}, 2 \mathrm{H}), 7.36-7.47(\mathrm{~m}, 4 \mathrm{H})$, 7.67-7.71 (m, 2H), $7.76(\mathrm{~s}, 1 \mathrm{H}) ;{ }^{13} \mathrm{C}$ NMR $\left(75 \mathrm{MHz}, \mathrm{CDCl}_{3}\right) \delta 24.36$ $\left(\mathrm{NCH}_{2} \mathrm{CH}_{2} \mathrm{CH}_{2}\right), 26.15\left(\mathrm{NCH}_{2} \mathrm{CH}_{2} \mathrm{CH}_{2} \times 2\right), 55.34\left(\mathrm{NCH}_{2} \mathrm{CH}_{2} \mathrm{CH}_{2} \times 2\right)$, $57.86\left(\mathrm{NCH}_{2} \mathrm{CH}_{2} \mathrm{O}\right), 66.94\left(\mathrm{OCH}_{2} \mathrm{CH}_{2} \mathrm{~N}\right), 101.34(\mathrm{CH}), 113.44(\mathrm{CH})$, $113.59(\mathrm{C}), 125.05(\mathrm{C}), 128.63(\mathrm{CH}), 129.01(\mathrm{CH}), 135.27(\mathrm{C}), 140.22$ $(\mathrm{CH}), 155.50(\mathrm{C}), 161.12(\mathrm{C}), 162.11(\mathrm{CO}) ; v_{\max }\left(\mathrm{KBr}, \mathrm{cm}^{-1}\right) 1721,1609$ 1268, 783; ES-MS $(m / z) 350[\mathrm{M}+\mathrm{H}]^{+}$. Found $\mathrm{C} 75.69 \%, \mathrm{H} 6.60 \%, \mathrm{~N}$ 4.04\%; $\mathrm{C}_{22} \mathrm{H}_{23} \mathrm{NO}_{3}$ requires C 75.62\%, $\mathrm{H} 6.63 \%, \mathrm{~N} 4.01 \%$.

3-(2-Chlorophenyl)-7-(2-diisopropylaminoethoxy) -2H-1benzopyran-2-one (3ba): Yield $81 \%$; mp $133-135^{\circ} \mathrm{C}$; ${ }^{1} \mathrm{H}$ NMR $(300$ $\left.\mathrm{MHz} \mathrm{CDCl}_{3}\right) \delta 1.07(\mathrm{~d}, 12 \mathrm{H}, J=6.5 \mathrm{~Hz}), 2.88(\mathrm{t}, 2 \mathrm{H}, J=7.5 \mathrm{~Hz}), 3.07$ (sep, $2 \mathrm{H}, J=6.5 \mathrm{~Hz}), 3.99(\mathrm{t}, 2 \mathrm{H}, J=6.6 \mathrm{~Hz}), 6.85-6.89(\mathrm{~m}, 2 \mathrm{H}), 7.32-7.36(\mathrm{~m}$, $2 \mathrm{H}), 7.39-7.43(\mathrm{~m}, 2 \mathrm{H}), 7.47-7.50(\mathrm{~m}, 1 \mathrm{H}), 7.69(\mathrm{~s}, 1 \mathrm{H}) ;{ }^{13} \mathrm{C}$ NMR $(50$ $\left.\mathrm{MHz}, \mathrm{CDCl}_{3}\right) \delta 21.08\left(\mathrm{CHCH}_{3} \times 4\right), 44.38\left(\mathrm{NCH}_{2}\right), 49.97\left(\mathrm{CH}_{3} \mathrm{CH} \times 2\right)$, $70.12\left(\mathrm{OCH}_{2}\right), 101.63(\mathrm{CH}), 112.80(\mathrm{C}), 113.33(\mathrm{CH}), 123.55(\mathrm{C})$, $127.01(\mathrm{CH}), 129.21(\mathrm{CH}), 129.98(\mathrm{CH}), 130.11(\mathrm{CH}), 131.75(\mathrm{CH})$, 134.01 (C), 134.33 (C), 143.02 (CH), 156.03 (C), 160.45 (C), 162.65 (CO); $v_{\max }\left(\mathrm{KBr}, \mathrm{cm}^{-1}\right) 1724,1612,1214,761$; ES-MS $(\mathrm{m} / z) 400[\mathrm{M}+\mathrm{H}]^{+}$. Found $\mathrm{C} 69.15 \%, \mathrm{H} 6.46 \%, \mathrm{~N} 3.48 \% ; \mathrm{C}_{23} \mathrm{H}_{26} \mathrm{ClNO}_{3}$ requires $\mathrm{C} 69.08 \%$, H 6.55\%, N 3.50\%.

3-(2-Chlorophenyl)-7-(2-pyrrolidin-1-yl-ethoxy)-

2H-1benzopyran-2-one (3bb): Yield 78\%; mp 95-97 ${ }^{\circ} \mathrm{C} ;{ }^{1} \mathrm{H}$ NMR $(300 \mathrm{MHz}$, $\left.\mathrm{CDCl}_{3}\right) \delta 1.84-1.85(\mathrm{~m}, 4 \mathrm{H}), 2.66(\mathrm{br} \mathrm{m}, 4 \mathrm{H}), 2.96(\mathrm{t}, 2 \mathrm{H}, J=5.6 \mathrm{~Hz})$, $4.19(\mathrm{t}, 2 \mathrm{H}, J=5.6 \mathrm{~Hz}), 6.89-6.93(\mathrm{~m}, 2 \mathrm{H}), 7.31-7.34(\mathrm{~m}, 2 \mathrm{H}), 7.39-7.43$ (m, 2H), 7.47-7.49 (m, 1H), $\left.7.68(\mathrm{~s}, 1 \mathrm{H}) ;{ }^{13} \mathrm{C} \mathrm{NMR} \mathrm{(50} \mathrm{MHz,} \mathrm{CDCl}_{3}\right)$ $\delta 23.72\left(\mathrm{NCH}_{2} \mathrm{CH}_{2} \times 2\right), 54.95\left(\mathrm{NCH}_{2} \times 3\right), 67.94\left(\mathrm{OCH}_{2}\right), 101.54(\mathrm{CH})$, $111.93(\mathrm{C}), 113.46(\mathrm{CH}), 123.69(\mathrm{C}), 127.00(\mathrm{CH}), 129.21(\mathrm{CH}), 129.99$ $(\mathrm{CH}), 130.08(\mathrm{CH}), 131.71(\mathrm{CH}), 133.96(\mathrm{C}), 134.25(\mathrm{C}), 142.97(\mathrm{CH})$, $155.94(\mathrm{C}), 160.39(\mathrm{C}), 162.40(\mathrm{CO}) ; v_{\max }\left(\mathrm{KBr}, \mathrm{cm}^{-1}\right) 1725,1613,1216$, 
Citation: Shailesh K, Devdutt C, Devi Prasad S (2017) Synthesis and Antimicrobial Activity of Novel 3,7-Disubstituted 2H-1-Benzopyran-2-Ones. Med Chem (Los Angeles) 7: 857-864. doi: 10.4172/2161-0444.1000442

761; ES-MS $(m / z) 370[\mathrm{M}+\mathrm{H}]^{+}$. Found C 68.31\%, H 5.40\%, N 3.77\%; $\mathrm{C}_{21} \mathrm{H}_{20} \mathrm{ClNO}_{3}$ requires $\mathrm{C} 68.20 \%, \mathrm{H} 5.45 \%, \mathrm{~N} 3.79 \%$.

3-(2-Chlorophenyl)-7-(2-piperidin-1-yl-ethoxy)-2H-1benzopyran-2-one (3bc): Yield $75 \%$; mp $105-107^{\circ} \mathrm{C} ;{ }^{1} \mathrm{H}$ NMR $(300$ $\left.\mathrm{MHz}, \mathrm{CDCl}_{3}\right) \delta 1.46-1.47(\mathrm{~m}, 2 \mathrm{H}), 1.61-1.62(\mathrm{~m}, 4 \mathrm{H}), 2.51-2.52(\mathrm{~m}$, $4 \mathrm{H}), 2.81(\mathrm{t}, 2 \mathrm{H}, J=5.9 \mathrm{~Hz}), 4.18(\mathrm{t}, 2 \mathrm{H}, J=6.0 \mathrm{~Hz}), 6.88-6.90(\mathrm{~m}$, $2 \mathrm{H}), 7.31-7.36(\mathrm{~m}, 2 \mathrm{H}), 7.39-7.42(\mathrm{~m}, 2 \mathrm{H}), 7.46-7.49(\mathrm{~m}, 1 \mathrm{H}), 7.68$ (s, $1 \mathrm{H}) ;{ }^{13} \mathrm{C}$ NMR $\left(50 \mathrm{MHz}, \mathrm{CDCl}_{3}\right) \delta 24.35\left(\mathrm{NCH}_{2} \mathrm{CH}_{2} \mathrm{CH}_{2}\right), 26.13$ $\left(\mathrm{NCH}_{2} \mathrm{CH}_{2} \mathrm{CH}_{2} \times 2\right), 53.33\left(\mathrm{NCH}_{2} \mathrm{CH}_{2} \mathrm{CH}_{2} \times 2\right), 57.83\left(\mathrm{NCH}_{2}\right), 66.96$ $\left(\mathrm{OCH}_{2}\right), 101.57(\mathrm{CH}), 112.91(\mathrm{C}), 113.48(\mathrm{CH}), 123.6(\mathrm{C}), 127.00$ $(\mathrm{CH}), 129.20(\mathrm{CH}), 129.99(\mathrm{CH}), 130.10(\mathrm{CH}), 131.72(\mathrm{CH}), 133.98$ (C), $134.27(\mathrm{C}), 142.97(\mathrm{CH}), 155.97(\mathrm{C}), 160.40(\mathrm{C}), 162.44(\mathrm{CO}) ; v_{\max }$ $\left(\mathrm{KBr}, \mathrm{cm}^{-1}\right) 1721,1609,1122,777$; ES-MS $(\mathrm{m} / z) 384[\mathrm{M}+\mathrm{H}]^{+}$. Found C $68.91 \%, \mathrm{H} 5.64 \%, \mathrm{~N} 3.63 \% ; \mathrm{C}_{22} \mathrm{H}_{22} \mathrm{ClNO}_{3}$ requires $\mathrm{C} 68.83 \%$, $\mathrm{H} 5.78 \%$, N 3.65\%.

7-(2-Dimethylaminoethoxy)-3-(2-methoxy phenyl)-2H-1benzopyran-2-one (3ca): Yield 70\%; viscous liquid; ${ }^{1} \mathrm{H}$ NMR (300 $\left.\mathrm{MHz} \mathrm{CDCl}_{3}\right) \delta 2.36(\mathrm{~s}, 6 \mathrm{H}), 2.77(\mathrm{t}, 2 \mathrm{H}, J=5.6 \mathrm{~Hz}), 3.82(\mathrm{~s}, 3 \mathrm{H}), 4.13$ $(\mathrm{t}, 2 \mathrm{H}, J=5.6 \mathrm{~Hz}), 6.86-6.89(\mathrm{~m}, 2 \mathrm{H}), 6.96-7.04(\mathrm{~m}, 2 \mathrm{H}), 7.34-7.39(\mathrm{~m}$, $3 \mathrm{H}), 7.67(\mathrm{~s}, 1 \mathrm{H}) ;{ }^{13} \mathrm{C} \mathrm{NMR}\left(75 \mathrm{MHz}, \mathrm{CDCl}_{3}\right) \delta 46.01\left(\mathrm{NCH}_{3} \times 2\right), 55.91$ $\left(\mathrm{OCH}_{3}\right), 58.13\left(\mathrm{NCH}_{2}\right), 66.72\left(\mathrm{OCH}_{2}\right), 101.29(\mathrm{CH}), 111.51(\mathrm{CH})$, $113.07(\mathrm{CH}), 113.41(\mathrm{C}), 120.73(\mathrm{CH}), 123.14(\mathrm{C}), 124.56(\mathrm{C}), 128.85$ (CH), $130.06(\mathrm{CH}), 131.01(\mathrm{CH}), 141.98(\mathrm{CH}), 155.55(\mathrm{C}), 157.44(\mathrm{C})$, $160.72(\mathrm{C}), 161.79(\mathrm{CO}) ; v_{\max }\left(\mathrm{KBr}, \mathrm{cm}^{-1}\right) 1727,1614,1266,738$; ES-MS $(m / z) 340[\mathrm{M}+\mathrm{H}]^{+}$. Found $\mathrm{C} 70.86 \%, \mathrm{H} 6.19 \%, \mathrm{~N} 4.12 \% ; \mathrm{C}_{20} \mathrm{H}_{21} \mathrm{NO}_{4}$ requires $\mathrm{C} 70.78 \%, \mathrm{H} 6.25 \%, \mathrm{~N} 4.13 \%$.

3-(2-Methoxyphenyl)-7-(2-pyrrolidin-1-yl-ethoxy)-2H-1benzopyran-2-one (3cb): Yield 72\%; viscous liquid; ${ }^{1} \mathrm{H}$ NMR $(300$ $\left.\mathrm{MHz}, \mathrm{CDCl}_{3}\right) \delta 1.84-1.86(\mathrm{~m}, 4 \mathrm{H}), 2.64-2.68(\mathrm{~m}, 4 \mathrm{H}), 2.96(\mathrm{t}, 2 \mathrm{H}, J=5.8$ $\mathrm{Hz}), 3.83(\mathrm{~s}, 3 \mathrm{H}), 4.19(\mathrm{t}, 2 \mathrm{H}, J=5.8 \mathrm{~Hz}), 6.87-6.89(\mathrm{~m}, 2 \mathrm{H}), 6.97-7.04$ (m, 2H), 7.34-7.39 (m, 3H), $7.67(\mathrm{~s}, 1 \mathrm{H}) ;{ }^{13} \mathrm{C} \mathrm{NMR}\left(75 \mathrm{MHz}, \mathrm{CDCl}_{3}\right) \delta$ 23.72, $\left(\mathrm{NCH}_{2} \mathrm{CH}_{2} \times 2\right), 54.97\left(\mathrm{NCH}_{2} \times 3\right), 55.99(\mathrm{OCH} 3), 67.84(\mathrm{OCH} 2)$, $101.44(\mathrm{CH}), 111,58(\mathrm{CH}), 113.12(\mathrm{CH}), 113.48(\mathrm{C}), 120.82(\mathrm{CH})$, $123.24(\mathrm{C}), 124.64(\mathrm{C}), 128.91(\mathrm{CH}), 130.14(\mathrm{CH}), 131.09(\mathrm{CH}), 142.04$ $(\mathrm{CH}), 155.65(\mathrm{C}), 157.52(\mathrm{C}), 160.83(\mathrm{C}), 161.86(\mathrm{CO}) ; v_{\max }(\mathrm{KBr}, \mathrm{cm}$ 1) $2361,1723,1613,1216,761$; ES-MS $(\mathrm{m} / z) 366[\mathrm{M}+\mathrm{H}]^{+}$. Found C $72.45 \%, \mathrm{H} 6.24 \%$, N 3.85\%; $\mathrm{C}_{22} \mathrm{H}_{23} \mathrm{NO}_{4}$ requires $\mathrm{C} 72.31 \%, \mathrm{H} 6.34 \%$, N $3.83 \%$.

7-(2-Diethylaminoethoxy)-3-(2-methoxyphenyl)

$-2 \mathrm{H}-1-$ benzopyran-2-one $(3 \mathrm{cc})$ : Yield $76 \%$; mp $113-115^{\circ} \mathrm{C}$; ${ }^{1} \mathrm{H}$ NMR $(300$ $\left.\mathrm{MHz}, \mathrm{CDCl}_{3}\right) \delta 1.09(\mathrm{t}, 6 \mathrm{H}, J=7.2 \mathrm{~Hz}), 2.66(\mathrm{q}, 4 \mathrm{H}, J=7.2 \mathrm{~Hz}), 2.91(\mathrm{t}$, $2 \mathrm{H}, J=6.1 \mathrm{~Hz}), 3.83(\mathrm{~s}, 3 \mathrm{H}), 4.12(\mathrm{t}, 2 \mathrm{H}, J=6.1 \mathrm{~Hz}), 6.84-6.88(\mathrm{~m}, 2 \mathrm{H})$, 6.97-7.04 (m, 2H), 7.34-7.39 (m, 3H), $7.67(\mathrm{~s}, 1 \mathrm{H}) ;{ }^{13} \mathrm{C} \mathrm{NMR}(100 \mathrm{MHz}$, $\left.\mathrm{CDCl}_{3}\right) \delta 11.99\left(\mathrm{NCH}_{2} \mathrm{CH}_{3} \times 2\right), 48.07\left(\mathrm{NCH}_{2} \times 3\right), 55.96\left(\mathrm{OCH}_{3}\right), 67.45$ $\left(\mathrm{OCH}_{2}\right), 101.36(\mathrm{CH}), 111.55(\mathrm{CH}), 113.09(\mathrm{CH}), 113.39(\mathrm{C}), 120.79$ $(\mathrm{CH}), 123.19(\mathrm{C}), 124.62(\mathrm{C}), 128.89(\mathrm{CH}), 130.12(\mathrm{CH}), 131.07(\mathrm{CH})$, $142.05(\mathrm{CH}), 155.62(\mathrm{C}), 157.50(\mathrm{C}), 160.83(\mathrm{C}), 161.92(\mathrm{CO}) ; v_{\max }(\mathrm{KBr}$, $\left.\mathrm{cm}^{-1}\right)$ 2361, 1726, 1610, 1265, 740; ES-MS $(\mathrm{m} / z) 368[\mathrm{M}+\mathrm{H}]^{+}$. Found C $72.05 \%, \mathrm{H} 6.69 \%, \mathrm{~N} 3.84 \%$; $\mathrm{C}_{22} \mathrm{H}_{25} \mathrm{NO}_{4}$ requires C $71.91 \%, \mathrm{H} 6.86 \%, \mathrm{~N}$ $3.81 \%$.

3-(3,4-Dimethoxyphenyl)-7-(2-dimethylaminoethoxy) 2H-1benzopyran-2-one (3da): Yield $80 \%$; mp $120-121^{\circ} \mathrm{C} ;{ }^{1} \mathrm{H}$ NMR $(300$ $\left.\mathrm{MHz}, \mathrm{CDCl}_{3}\right) \delta 2.36(\mathrm{~s}, 6 \mathrm{H}), 2.78(\mathrm{t}, 2 \mathrm{H}, J=5.6 \mathrm{~Hz}), 3.92(\mathrm{~s}, 3 \mathrm{H}), 3.94$ $(\mathrm{s}, 3 \mathrm{H}), 4.14(\mathrm{t}, 2 \mathrm{H}, J=5.6 \mathrm{~Hz}), 6.87-6.94(\mathrm{~m}, 3 \mathrm{H}), 7.24-7.29(\mathrm{~m}, 2 \mathrm{H})$, $7.42(\mathrm{~d}, 1 \mathrm{H}, J=8.6 \mathrm{~Hz}), 7.72(\mathrm{~s}, 1 \mathrm{H}) ;{ }^{13} \mathrm{C} \mathrm{NMR}\left(75 \mathrm{MHz}, \mathrm{CDCl}_{3}\right) \delta 46.09$ $\left(\mathrm{NCH}_{3} \times 2\right), 56.15\left(\mathrm{OCH}_{3}\right), 56.21\left(\mathrm{OCH}_{3}\right), 58.21\left(\mathrm{NCH}_{2}\right), 66.82\left(\mathrm{OCH}_{2}\right)$, $101.17(\mathrm{CH}), 111.26(\mathrm{CH}), 111.99(\mathrm{CH}), 113.40(\mathrm{CH}), 113.67(\mathrm{C})$, $121.21(\mathrm{CH}), 124.65(\mathrm{C}), 127.98(\mathrm{C}), 128.80(\mathrm{CH}), 139.13(\mathrm{CH}), 148.90$ (C), $149.61(\mathrm{C}), 155.19(\mathrm{C}), 161.20(\mathrm{C}), 161.83(\mathrm{CO}) ; v_{\max }\left(\mathrm{KBr}, \mathrm{cm}^{-1}\right)$
$2361,1720,1611,1216,1027,761$; ES-MS $(m / z) 370[\mathrm{M}+\mathrm{H}]^{+}$. Found $\mathrm{C} 68.37 \%, \mathrm{H} 6.30 \%, \mathrm{~N} 3.82 \%$; $\mathrm{C}_{21} \mathrm{H}_{23} \mathrm{NO}_{5}$ requires $\mathrm{C} 68.28 \%, \mathrm{H} 6.28 \%$, N $3.79 \%$.

3-(3,4-Dimethoxyphenyl)-7-(2-pyrrolidin-1-yl-ethoxy)-2H-1benzopyran-2-one (3db): Yield 78\%; mp $90-91^{\circ} \mathrm{C}$; ${ }^{1} \mathrm{H}$ NMR $(300 \mathrm{MHz}$, $\left.\mathrm{CDCl}_{3}\right) \delta 1.81-1.86(\mathrm{~m}, 4 \mathrm{H}), 2.61-2.68(\mathrm{~m}, 4 \mathrm{H}), 2.95(\mathrm{t}, 2 \mathrm{H}, J=5.8 \mathrm{~Hz})$, $3.92(\mathrm{~s}, 3 \mathrm{H}), 3.94(\mathrm{~s}, 3 \mathrm{H}), 4.19(\mathrm{t}, 2 \mathrm{H}, J=5.8 \mathrm{~Hz}), 6.87-6.95(\mathrm{~m}, 3 \mathrm{H})$, 7.24-7.29 (m, 2H), $7.42(\mathrm{~d}, 1 \mathrm{H}, J=8.4 \mathrm{~Hz}), 7.72(\mathrm{~s}, 1 \mathrm{H}) ;{ }^{13} \mathrm{C}$ NMR $(75$ $\left.\mathrm{MHz}, \mathrm{CDCl}_{3}\right) \delta 23.67\left(\mathrm{NCH}_{2} \mathrm{CH}_{2} \times 2\right), 54.94\left(\mathrm{NCH}_{2} \times 3\right), 56.13\left(\mathrm{OCH}_{3}\right)$, $56.17\left(\mathrm{OCH}_{3}\right), 67.86\left(\mathrm{OCH}_{2}\right), 101.20(\mathrm{CH}), 111.16(\mathrm{CH}), 111.88(\mathrm{CH})$, $113.35(\mathrm{CH}), 113.63(\mathrm{C}), 121.17(\mathrm{CH}), 124.59(\mathrm{C}), 127.94(\mathrm{C}), 128.81$ $(\mathrm{CH}), 139.17(\mathrm{CH}), 148.83(\mathrm{C}), 149.54(\mathrm{C}), 155.16(\mathrm{C}), 161.24(\mathrm{C})$, $161.81(\mathrm{CO}) ; v_{\max }\left(\mathrm{KBr}, \mathrm{cm}^{-1}\right) 2359,1724,1612,1210,1012740$; ES-MS $(\mathrm{m} / \mathrm{z}) 396[\mathrm{M}+\mathrm{H}]^{+}$. Found C 69.97\%, H 6.29\%, N 3.52\%; $\mathrm{C}_{23} \mathrm{H}_{25} \mathrm{NO}_{5}$ requires C 69.86\%, H 6.37\%, N 3.54\%.

3-(3,4-Dimethoxyphenyl)-7-(2-piperidin-1-yl-ethoxy)-2H1-benzopyran-2-one (3dc): Yield 78\%; mp 96-98 ${ }^{\circ} \mathrm{C} ;{ }^{1} \mathrm{H}$ NMR (400 $\left.\mathrm{MHz}, \mathrm{CDCl}_{3}\right) \delta 1.45-1.49(\mathrm{~m}, 2 \mathrm{H}), 1.60-1.66(\mathrm{~m}, 4 \mathrm{H}), 2.51-2.54(\mathrm{~m}$, $4 \mathrm{H}), 2.81(\mathrm{t}, 2 \mathrm{H}, J=5.9 \mathrm{~Hz}), 3.92(\mathrm{~s}, 3 \mathrm{H}), 3.94(\mathrm{~s}, 3 \mathrm{H}), 4.18(\mathrm{t}, 2 \mathrm{H}, J=5.9$ $\mathrm{Hz}), 6.86-6.89(\mathrm{~m}, 2 \mathrm{H}), 6.93(\mathrm{~d}, 1 \mathrm{H}, J=8.3 \mathrm{~Hz}), 7.24-7.29(\mathrm{~m}, 2 \mathrm{H}), 7.42$ (d, $1 \mathrm{H}, J=8.4 \mathrm{~Hz}), 7.72(\mathrm{~s}, 1 \mathrm{H}) ;{ }^{13} \mathrm{C} \mathrm{NMR}\left(75 \mathrm{MHz}, \mathrm{CDCl}_{3}\right) \delta 24.33$ $\left(\mathrm{NCH}_{2} \mathrm{CH}_{2} \mathrm{CH}_{2}\right), 26.09\left(\mathrm{NCH}_{2} \mathrm{CH}_{2} \mathrm{CH}_{2} \times 2\right), 53.31\left(\mathrm{NCH}_{2} \mathrm{CH}_{2} \mathrm{CH}_{2} \times 2\right)$, $56.19\left(\mathrm{OCH}_{3}\right), 56.24\left(\mathrm{OCH}_{3}\right), 57.84\left(\mathrm{NCH}_{2}\right), 66.87\left(\mathrm{OCH}_{2}\right), 101.33$ $(\mathrm{CH}), 111.29(\mathrm{CH}), 112.03(\mathrm{CH}), 113.39(\mathrm{CH}), 113.69(\mathrm{CH}), 121.23$ $(\mathrm{CH}), 124.68(\mathrm{C}), 128.03(\mathrm{C}), 128.82(\mathrm{CH}), 139.16(\mathrm{CH}), 148.94(\mathrm{C})$, $149.65(\mathrm{C}), 155.25(\mathrm{C}), 161.26(\mathrm{C}), 161.86(\mathrm{CO}) ; v_{\max }\left(\mathrm{KBr}, \mathrm{cm}^{-1}\right) 1723$, 1610, 1210, 1009 738; ES-MS $(\mathrm{m} / \mathrm{z}) 410[\mathrm{M}+\mathrm{H}]^{+}$. Found C 70.52\%, H $6.69 \%, \mathrm{~N} 3.46 \% ; \mathrm{C}_{24} \mathrm{H}_{27} \mathrm{NO}_{5}$ requires C 70.40\%, H 6.65\%, N 3.42\%.

7-(2-Diisopropylaminoethoxy)-3-(3,4-dimethoxy-phenyl) -2H-1-benzopyran-2-one (3dd): Yield $88 \%$; mp $113-115^{\circ} \mathrm{C} ;{ }^{1} \mathrm{H}$ NMR $\left(300 \mathrm{MHz}, \mathrm{CDCl}_{3}\right) \delta 1.06(\mathrm{~d}, 12 \mathrm{H}, J=6.5 \mathrm{~Hz}), 2.86(\mathrm{t}, 2 \mathrm{H}, J=7.3 \mathrm{~Hz})$, 3.06 (sep, $2 \mathrm{H}, J=6.5 \mathrm{~Hz}$ ), $3.92(\mathrm{~s}, 3 \mathrm{H}), 3.95$ (s, 3H), 3.97 (t, 2H, $J=7.2$ $\mathrm{Hz}), 6.84-6.87$ (m, 2H), 6.93 (d, 1H, J=8.4 Hz), 7.24-7.29 (m, 2H), 7.42 $(\mathrm{d}, 1 \mathrm{H}, J=9.3 \mathrm{~Hz}), 7.72(\mathrm{~s}, 1 \mathrm{H}) ;{ }^{13} \mathrm{C}$ NMR $\left(50 \mathrm{MHz}, \mathrm{CDCl}_{3}\right) \delta 21.09$ $\left(\mathrm{CHCH}_{3} \times 4\right), 44.37\left(\mathrm{NCH}_{2}\right), 49.90\left(\mathrm{CH}_{3} \mathrm{CH} \times 2\right), 56.18\left(\mathrm{OCH}_{3}\right), 56.24$ $\left(\mathrm{OCH}_{3}\right), 70.09\left(\mathrm{OCH}_{2}\right), 101.33(\mathrm{CH}), 111.29(\mathrm{CH}), 112.04(\mathrm{CH}), 113.24$ $(\mathrm{CH}), 113.53(\mathrm{C}), 121.22(\mathrm{CH}), 124.52(\mathrm{C}), 128.08(\mathrm{C}), 128.82(\mathrm{CH})$, $139.22(\mathrm{CH}), 148.93(\mathrm{C}), 149.61(\mathrm{C}), 155.30$ (C), $161.31(\mathrm{C}), 162.09$ (CO); $v_{\text {max }}\left(\mathrm{KBr}, \mathrm{cm}^{-1}\right) 2361,1716,1612,1216,1025$ 761; ES-MS $(\mathrm{m} / z)$ $426[\mathrm{M}+\mathrm{H}]^{+}$; HRMS-EI: found 425.2234, calcuated 425.2202. Found C $70.69 \%, \mathrm{H} 7.23 \%, \mathrm{~N} 3.24 \% ; \mathrm{C}_{25} \mathrm{H}_{31} \mathrm{NO}_{5}$ requires C 70.57\%, H 7.34\%, N $3.29 \%$.

3-Naphthalen-1-yl-7-(2-piperidin-1-yl-ethoxy)

2H-1benzopyran-2-one (3ea): Yield $87 \%$; mp $86-88^{\circ} \mathrm{C}$; ${ }^{1} \mathrm{H}$ NMR $(300 \mathrm{MHz}$, $\left.\mathrm{CDCl}_{3}\right) \delta 1.41-1.51(\mathrm{~m}, 2 \mathrm{H}), 1.60-1.68(\mathrm{~m}, 4 \mathrm{H}), 2.53-2.56(\mathrm{~m}, 4 \mathrm{H}), 2.83$ $(\mathrm{t}, 2 \mathrm{H}, J=5.9 \mathrm{~Hz}), 4.21(\mathrm{t}, 2 \mathrm{H}, J=5.9 \mathrm{~Hz}), 6.89-6.93(\mathrm{~m}, 2 \mathrm{H}), 7.42(\mathrm{~d}, 1 \mathrm{H}$, $J=8.5 \mathrm{~Hz}), 7.46-7.53(\mathrm{~m}, 4 \mathrm{H}), 7.75(\mathrm{~s}, 1 \mathrm{H}), 7.78-7.81(\mathrm{~m}, 1 \mathrm{H}), 7.88-7.92$ $(\mathrm{m}, 2 \mathrm{H}) ;{ }^{13} \mathrm{C}$ NMR $\left(75 \mathrm{MHz}, \mathrm{CDCl}_{3}\right) \delta 24.32\left(\mathrm{NCH}_{2} \mathrm{CH}_{2} \mathrm{CH}_{2}\right), 26.09$ $\left(\mathrm{NCH}_{2} \mathrm{CH}_{2} \mathrm{CH}_{2} \times 2\right), 55.32\left(\mathrm{NCH}_{2} \mathrm{CH}_{2} \mathrm{CH}_{2} \times 2\right), 57.82\left(\mathrm{NCH}_{2} \mathrm{CH}_{2} \mathrm{O}\right)$, $66.90\left(\mathrm{OCH}_{2} \mathrm{CH}_{2} \mathrm{~N}\right), 101.53(\mathrm{CH}), 113.20(\mathrm{C}), 113.43(\mathrm{CH}), 124.85$ (C), $125.43(\mathrm{CH}), 125.48(\mathrm{CH}), 126.21(\mathrm{CH}), 126.58(\mathrm{CH}), 127.91$ $(\mathrm{CH}), 128.71(\mathrm{CH}), 129.04(\mathrm{CH}), 129.33(\mathrm{CH}), 131.87(\mathrm{C}), 133.20(\mathrm{C})$, $133.89(\mathrm{C}), 143.12(\mathrm{CH}), 155.94(\mathrm{C}), 161.35(\mathrm{C}), 162.24(\mathrm{C}) ; v_{\max }(\mathrm{KBr}$, $\left.\mathrm{cm}^{-1}\right)$ 1710, 1612, 1215, 1010 777; ES-MS $(\mathrm{m} / \mathrm{z}) 400[\mathrm{M}+\mathrm{H}]^{+}$. Found C 78.30\%, H 6.24\%, N 3.48\%; $\mathrm{C}_{26} \mathrm{H}_{25} \mathrm{NO}_{3}$ requires C 78.18\%, $\mathrm{H} 6.31 \%, \mathrm{~N}$ $3.51 \%$.

7-(2-Diethylaminoethoxy) - 3-naphthalen-1-yl-2 H- 1 benzopyran-2-one (3eb): Yield $89 \%$; mp $78-80^{\circ} \mathrm{C}$; ${ }^{1} \mathrm{H} \mathrm{NMR}(300 \mathrm{MHz}$, $\mathrm{CDCl}_{3}$ ) $\delta 1.09(\mathrm{t}, 6 \mathrm{H}, J=7.1 \mathrm{~Hz}$ ), 2.67 (quart, $4 \mathrm{H}, J=7.1 \mathrm{~Hz}$ ), $2.93(\mathrm{t}$, 
$2 \mathrm{H}, J=6.1 \mathrm{~Hz}), 4.15(\mathrm{t}, 2 \mathrm{H}, J=6.1 \mathrm{~Hz}), 6.89-6.93(\mathrm{~m}, 2 \mathrm{H}), 7.41-7.54(\mathrm{~m}$, $5 \mathrm{H})$, 7.75-7.81 (m, 2H), 7.89-7.91 (m, 2H); ${ }^{13} \mathrm{C} \mathrm{NMR}\left(75 \mathrm{MHz}, \mathrm{CDCl}_{3}\right)$ $\delta 11.89\left(\mathrm{NCH}_{2} \mathrm{CH}_{3} \times 2\right), 48.08\left(\mathrm{NCH}_{2} \mathrm{CH}_{3} \times 2\right), 51.67\left(\mathrm{NCH}_{2} \mathrm{CH}_{2} \mathrm{O}\right)$, $67.42\left(\mathrm{OCH}_{2} \mathrm{CH}_{2} \mathrm{~N}\right), 101.52(\mathrm{CH}), 113.24(\mathrm{C}), 113.39(\mathrm{CH}), 124.88$ (C), $125.45(\mathrm{CH}), 125.51(\mathrm{CH}), 126.23(\mathrm{CH}), 126.61(\mathrm{CH}), 127.93$ $(\mathrm{CH}), 128.73(\mathrm{CH}), 129.07(\mathrm{CH}), 129.35(\mathrm{CH}), 131.88(\mathrm{C}), 133.21(\mathrm{C})$, $133.91(\mathrm{C}), 143.16(\mathrm{CH}), 155.95(\mathrm{C}), 161.38(\mathrm{C}), 162.24(\mathrm{C}) ; v_{\max }(\mathrm{KBr}$, $\left.\mathrm{cm}^{-1}\right)$ 1710, 1614, 1216, 1009 780; ES-MS $(\mathrm{m} / z) 388[\mathrm{M}+\mathrm{H}]^{+}$. Found C $77.61 \%, \mathrm{H} 6.46 \%, \mathrm{~N} 3.58 \% ; \mathrm{C}_{25} \mathrm{H}_{25} \mathrm{NO}_{3}$ requires C $77.49 \%, \mathrm{H} 6.50 \%, \mathrm{~N}$ $3.61 \%$

3-(3,4-Dichlorophenyl)-7-(2-piperidin-1-yl-ethoxy) 2H-1benzopyran-2-one (3fa): Yield $84 \%$; mp $110-112^{\circ} \mathrm{C}$; ${ }^{1} \mathrm{H}$ NMR $(300$ $\left.\mathrm{MHz}, \mathrm{CDCl}_{3}\right) \delta 1.49-1.53(\mathrm{~m}, 2 \mathrm{H}), 1.63-1.70(\mathrm{~m}, 4 \mathrm{H}), 2.57-2.59(\mathrm{~m}$, $4 \mathrm{H}), 2.86(\mathrm{t}, 2 \mathrm{H}, J=5.8 \mathrm{~Hz}), 4.21(\mathrm{t}, 2 \mathrm{H}, J=5.8 \mathrm{~Hz}), 6.87-6.90(\mathrm{~m}, 2 \mathrm{H})$, 7.33-7.36 (m, 2H), 7.40-7.43 (m, 1H), $7.52(\mathrm{~d}, 1 \mathrm{H}, J=1.7 \mathrm{~Hz}), 7.69$ (s, 1H); ${ }^{13} \mathrm{C} \mathrm{NMR}\left(75 \mathrm{MHz}, \mathrm{CDCl}_{3}\right) \delta 24.24\left(\mathrm{NCH}_{2} \mathrm{CH}_{2} \mathrm{CH}_{2}\right), 25.89$ $\left(\mathrm{NCH}_{2} \mathrm{CH}_{2} \mathrm{CH}_{2} \times 2\right), 55.28\left(\mathrm{NCH}_{2} \mathrm{CH}_{2} \mathrm{CH}_{2} \times 2\right), 57.77\left(\mathrm{NCH}_{2} \mathrm{CH}_{2} \mathrm{O}\right)$, $66.67\left(\mathrm{OCH}_{2} \mathrm{CH}_{2} \mathrm{~N}\right), 101.56(\mathrm{CH}), 112.77(\mathrm{C}), 113.59(\mathrm{CH}), 122.49$ $(\mathrm{C}), 127.39(\mathrm{CH}), 129.30(\mathrm{CH}), 129.99(\mathrm{CH}), 132.54(\mathrm{CH}), 132.73(\mathrm{C})$, 134.77 (C), 135.27 (C), 143.29 (CH), 155.97 (C), 160.28 (C), 162.53 (C); $v_{\max }\left(\mathrm{KBr}, \mathrm{cm}^{-1}\right) 1724,1613,1216,761$; ES-MS $(\mathrm{m} / z) 418[\mathrm{M}+\mathrm{H}]^{+}$. Found $\mathrm{C} 63.28 \%, \mathrm{H} 5.02 \%, \mathrm{~N} 3.34 \% ; \mathrm{C}_{22} \mathrm{H}_{21} \mathrm{Cl}_{2} \mathrm{NO}_{3}$ requires $\mathrm{C} 63.17 \%$, H 5.06\%, N 3.35\%.

3-(3,4-Dichlorophenyl)-7-(2-diethylaminoethoxy)

$-2 \mathrm{H}-1-$ benzopyran-2-one (3fb): Yield $78 \% ; \mathrm{mp} 66-68^{\circ} \mathrm{C} ;{ }^{1} \mathrm{H}$ NMR $(300 \mathrm{MHz}$ $\left.\mathrm{CDCl}_{3}\right) \delta 1.08(\mathrm{t}, 6 \mathrm{H}, J=7.1 \mathrm{~Hz}), 2.65$ (quart, $4 \mathrm{H}, J=7.1 \mathrm{~Hz}$ ), $2.91(\mathrm{t}$, $2 \mathrm{H}, J=6.1 \mathrm{~Hz}), 4.12(\mathrm{t}, 2 \mathrm{H}, J=6.1 \mathrm{~Hz}), 6.88-6.92(\mathrm{~m}, 2 \mathrm{H}), 7.29-7.38(\mathrm{~m}$, $2 \mathrm{H}), 7.40-7.43(\mathrm{~m}, 1 \mathrm{H}), 7.50(\mathrm{~d}, 1 \mathrm{H}, J=1.8 \mathrm{~Hz}), 7.68(\mathrm{~s}, 1 \mathrm{H}) ;{ }^{13} \mathrm{C} \mathrm{NMR}$ $\left(50 \mathrm{MHz}, \mathrm{CDCl}_{3}\right) \delta 11.98\left(\mathrm{NCH}_{2} \mathrm{CH}_{3} \times 2\right), 48.08\left(\mathrm{NCH}_{2} \mathrm{CH}_{3} \times 2\right), 51.72$ $\left(\mathrm{NCH}_{2}\right), 67.62\left(\mathrm{OCH}_{2}\right), 101.52(\mathrm{CH}), 112.70(\mathrm{C}), 113.59(\mathrm{CH}), 122.42$ (C), $127.37(\mathrm{CH}), 129.29(\mathrm{CH}), 129.96(\mathrm{CH}), 132.54(\mathrm{CH}), 132.77(\mathrm{C})$, 134.77 (C), $135.22(\mathrm{C}), 143.28(\mathrm{CH}), 156.00(\mathrm{C}), 160.23(\mathrm{C}), 162.68(\mathrm{C})$; $v_{\max }\left(\mathrm{KBr}, \mathrm{cm}^{-1}\right) 1723,1614,1216,1026763$; ES-MS $(\mathrm{m} / z) 406[\mathrm{M}+\mathrm{H}]^{+}$. Found C 62.20\%, $\mathrm{H} 5.27 \%, \mathrm{~N} 3.47 \% ; \mathrm{C}_{21} \mathrm{H}_{21} \mathrm{Cl}_{2} \mathrm{NO}_{3}$ requires $\mathrm{C} 62.08 \%$, H $5.21 \%$, N 3.45\%.

3-(4-Fluorophenyl)-7-(2-piperidin-1-yl-ethoxy)-

2H-1benzopyran-2-one (3ga): Yield 79\%; mp $124-126^{\circ} \mathrm{C}$; ${ }^{1} \mathrm{H}$ NMR $(300$ $\left.\mathrm{MHz}, \mathrm{CDCl}_{3}\right) \delta 1.44-1.49(\mathrm{~m}, 2 \mathrm{H}), 1.59-1.67(\mathrm{~m}, 4 \mathrm{H}), 2.51-2.54(\mathrm{~m}$, $4 \mathrm{H}), 2.81(\mathrm{t}, 2 \mathrm{H}, J=5.9 \mathrm{~Hz}), 4.18(\mathrm{t}, 2 \mathrm{H}, J=5.9 \mathrm{~Hz}), 6.86-6.91(\mathrm{~m}, 2 \mathrm{H})$, 7.09-7.15 (m, 2H), $7.43(\mathrm{~d}, 1 \mathrm{H}, J=8.6 \mathrm{~Hz}), 7.65-7.69(\mathrm{~m}, 2 \mathrm{H}), 7.73$ (s, $1 \mathrm{H}) ;{ }^{13} \mathrm{C}$ NMR $\left(50 \mathrm{MHz}, \mathrm{CDCl}_{3}\right) \delta 24.34\left(\mathrm{NCH}_{2} \mathrm{CH}_{2} \mathrm{CH}_{2}\right), 26.11$ $\left(\mathrm{NCH}_{2} \mathrm{CH}_{2} \mathrm{CH}_{2} \times 2\right), 55.33\left(\mathrm{NCH}_{2} \mathrm{CH}_{2} \mathrm{CH}_{2} \times 2\right), 57.84\left(\mathrm{NCH}_{2}\right), 66.93$ $\left(\mathrm{OCH}_{2}\right), 101.38(\mathrm{CH}), 113.48(\mathrm{C}), 113.54(\mathrm{CH}), 115.40(\mathrm{CH}), 115.83$ $(\mathrm{CH}), 124.02(\mathrm{C}), 129.01(\mathrm{CH}), 130.36(\mathrm{CH}), 130.53(\mathrm{CH}), 131.31(\mathrm{C})$, $140.07(\mathrm{CH}), 155.47(\mathrm{C}), 162.18(\mathrm{C}) ; v_{\max }\left(\mathrm{KBr}, \mathrm{cm}^{-1}\right) 1723,1616,1210$, 768; ES-MS $(m / z) 368[\mathrm{M}+\mathrm{H}]^{+}$. Found C 72.03\%, H 6.03\%, N 3.86\% ; $\mathrm{C}_{22} \mathrm{H}_{22} \mathrm{FNO}_{3}$ requires C 71.92\%, H 6.04\%, N 3.81\%.

7-(2-Diethylaminoethoxy)-3-(4-fluorophenyl)-

2H-1benzopyran-2-one (3gb): Yield $81 \%$; mp $95-97^{\circ} \mathrm{C}$; ${ }^{1} \mathrm{H}$ NMR $(300$ $\left.\mathrm{MHz}, \mathrm{CDCl}_{3}\right) \delta 1.09(\mathrm{t}, 6 \mathrm{H}, J=7.1 \mathrm{~Hz}), 2.66(\mathrm{q}, 4 \mathrm{H}, \mathrm{J}=7.1 \mathrm{~Hz}), 2.91$ $(\mathrm{t}, 2 \mathrm{H}, J=6.1 \mathrm{~Hz}), 4.12(\mathrm{t}, 2 \mathrm{H}, J=6.1 \mathrm{~Hz}), 6.86-6.90(\mathrm{~m}, 2 \mathrm{H}), 7.09-7.15$ $(\mathrm{m}, 2 \mathrm{H}), 7.42(\mathrm{~d}, 1 \mathrm{H}, J=8.3 \mathrm{~Hz}), 7.65-7.69(\mathrm{~m}, 2 \mathrm{H}), 7.73(\mathrm{~s}, 1 \mathrm{H}) ;{ }^{13} \mathrm{C}$ NMR $\left(75 \mathrm{MHz}, \mathrm{CDCl}_{3}\right) \delta 12.04\left(\mathrm{NCH}_{2} \mathrm{CH}_{3} \times 2\right), 48.13\left(\mathrm{NCH}_{2} \mathrm{CH}_{3} \times 2\right)$, $51.75\left(\mathrm{NCH}_{2}\right), 67.62\left(\mathrm{OCH}_{2}\right), 101.31(\mathrm{CH}), 113.44(\mathrm{C}), 113.51(\mathrm{CH})$, $115.46(\mathrm{CH}), 115.74(\mathrm{CH}), 123.97(\mathrm{C}), 129.00(\mathrm{CH}), 130.38(\mathrm{CH})$, $130.48(\mathrm{CH}), 131.25(\mathrm{C}), 140.07(\mathrm{CH}), 155.47(\mathrm{C}), 161.10(\mathrm{C}), 162.23$ (CO); $v_{\max }\left(\mathrm{KBr}, \mathrm{cm}^{-1}\right) 1723,1614,1210,770$; ES-MS $(\mathrm{m} / z) 356[\mathrm{M}+\mathrm{H}]^{+}$. Found C $71.02 \%, \mathrm{H} 6.29 \%, \mathrm{~N} 3.89 \% ; \mathrm{C}_{21} \mathrm{H}_{22} \mathrm{FNO}_{3}$ requires $\mathrm{C} 70.97 \%, \mathrm{H}$ $6.24 \%$, N 3.94\%.
3-(4-Chlorophenyl)-7-(2-piperidin-1-yl-ethoxy)-2H-1benzopyran-2-one (3ha): Yield $68 \%$; mp $118-120^{\circ} \mathrm{C} ;{ }^{1} \mathrm{H}$ NMR $(300$ $\left.\mathrm{MHz}, \mathrm{CDCl}_{3}\right) \delta 1.46-1.48(\mathrm{~m}, 2 \mathrm{H}), 1.61-1.63(\mathrm{~m}, 4 \mathrm{H}), 2.53(\mathrm{br} \mathrm{m}$, $4 \mathrm{H}), 2.81(\mathrm{t}, 2 \mathrm{H}, J=5.9 \mathrm{~Hz}), 4.18(\mathrm{t}, 2 \mathrm{H}, J=5.9 \mathrm{~Hz}), 6.86-6.90(\mathrm{~m}, 2 \mathrm{H})$, 7.39-7.44 (m, 3H), 7.63-7.66 (m, 2H), $7.75(\mathrm{~s}, 1 \mathrm{H}) ;{ }^{13} \mathrm{C} \mathrm{NMR}(75 \mathrm{MHz}$, $\left.\mathrm{CDCl}_{3}\right) \delta 24.31\left(\mathrm{NCH}_{2} \mathrm{CH}_{2} \mathrm{CH}_{2}\right), 26.08\left(\mathrm{NCH}_{2} \mathrm{CH}_{2} \mathrm{CH}_{2} \times 2\right), 55.32$ $\left(\mathrm{NCH}_{2} \mathrm{CH}_{2} \mathrm{CH}_{2} \times 2\right), 57.81\left(\mathrm{NCH}_{2} \mathrm{CH}_{2} \mathrm{O}\right), 66.88\left(\mathrm{OCH}_{2} \mathrm{CH}_{2} \mathrm{~N}\right), 101.33$ $(\mathrm{CH}), 113.38(\mathrm{CH}), 113.59(\mathrm{CH}), 123.74(\mathrm{C}), 128.83(\mathrm{CH}), 129.10$ $(\mathrm{CH}), 129.89(\mathrm{CH}), 133.64(\mathrm{C}), 134.63(\mathrm{C}), 140.31(\mathrm{CH}), 155.52(\mathrm{C})$, $160.91(\mathrm{C}), 162.28(\mathrm{C}) ; v_{\max }\left(\mathrm{KBr}, \mathrm{cm}^{-1}\right) 1721,1612,1198,763$; ES-MS $(m / z) 384[\mathrm{M}+\mathrm{H}]^{+}$. Found C 68.92\%, H 5.72\%, N 3.63\%; $\mathrm{C}_{22} \mathrm{H}_{22} \mathrm{ClNO}_{3}$ requires $\mathrm{C} 68.83 \%$, $\mathrm{H} 5.78 \%, \mathrm{~N} 3.65 \%$.

3-(4-Chlorophenyl)-7-(2-diethylaminoethoxy)-

2H-1benzopyran-2-one (3hb): Yield $65 \%$; mp $138-140^{\circ} \mathrm{C}$; ${ }^{1} \mathrm{H}$ NMR $(200$ $\mathrm{MHz}, \mathrm{CDCl}_{3}$ ) $\delta 1.09(\mathrm{t}, 6 \mathrm{H}, J=7.1 \mathrm{~Hz}$ ), 2.66 (quart, $4 \mathrm{H}, \mathrm{J}=7.1 \mathrm{~Hz}$ ), 2.92 $(\mathrm{t}, 2 \mathrm{H}, J=6.0 \mathrm{~Hz}), 4.14(\mathrm{t}, 2 \mathrm{H}, J=6.0 \mathrm{~Hz}), 6.86-6.94(\mathrm{~m}, 2 \mathrm{H}), 7.37-7.44$ $(\mathrm{m}, 3 \mathrm{H}), 7.64-7.69(\mathrm{~m}, 2 \mathrm{H}), 7.83(\mathrm{~s}, 1 \mathrm{H}) ;{ }^{13} \mathrm{C} \mathrm{NMR}\left(75 \mathrm{MHz}, \mathrm{CDCl}_{3}\right)$ $\delta 11.89\left(\mathrm{NCH}_{2} \mathrm{CH}_{3} \times 2\right), 47.92\left(\mathrm{NCH}_{2} \mathrm{CH}_{3} \times 2\right), 50.97\left(\mathrm{NCH}_{2}\right), 66.87$ $\left(\mathrm{OCH}_{2}\right), 101.36(\mathrm{CH}), 113.36(\mathrm{C}), 113.58(\mathrm{CH}), 123.73(\mathrm{C}), 128.83$ $(\mathrm{CH}), 129.10(\mathrm{CH}), 129.86(\mathrm{CH}), 133.56(\mathrm{C}), 134.61(\mathrm{C}), 140.32(\mathrm{CH})$ $155.50(\mathrm{C}), 161.01(\mathrm{C}), 162.30(\mathrm{CO}) ; v_{\max }\left(\mathrm{KBr}, \mathrm{cm}^{-1}\right) 1722,1614,1201$, 770; ES-MS $(m / z) 372[\mathrm{M}+\mathrm{H}]^{+}$. Found C 67.91\%, H 5.91\%, N 3.72\%; $\mathrm{C}_{21} \mathrm{H}_{22} \mathrm{ClNO}_{3}$ requires C 67.83\%, H 5.96\%, N 3.77\%.

\section{Biological activity}

Antimicrobial activity: All the synthesized compounds were screened for their antimicrobial activities. The bacterial and fungal strains were grown on nutrient agar at $37^{\circ} \mathrm{C}$. After $24 \mathrm{~h}$ of incubation, bacterial cells were suspended in normal saline containing Tween 20 at $0.05 \%$ at a concentration of approximately $1.0-2.0 \times 10^{7}$ cells $/ \mathrm{mL}$ by matching with $0.5 \mathrm{McF}$ arland standards. The activity of compounds was determined as per CLSI protocol using Mueller Hinton broth (Becton Dickinson, USA) in 96-well tissue culture plates. Proper growth control, drug control and the negative control were adjusted onto the plate. Compounds were dissolved in DMSO at a concentration of $1 \mathrm{mg} / \mathrm{mL}$ and $20 \mu \mathrm{L}$ of this was added to each well of 96-well tissue culture plate having $180 \mu \mathrm{L}$ Mueller Hinton broth. From here the solution was serially diluted resulting in two-fold dilution of the test compounds in subsequent wells. $100 \mu \mathrm{L}$ of Mc Farland matched bacterial suspension was diluted in $10 \mathrm{ml}$ of media and then $100 \mu \mathrm{L}$ of it was added in each well and kept for incubation. The maximum concentration of compounds tested was $50 \mu \mathrm{g} / \mathrm{mL}$. The micro-titer plates were incubated at $35^{\circ} \mathrm{C}$ in a moist, dark chamber and MICs were recorded spectrophotometrically after $24 \mathrm{~h}$ using SOFT max Pro 4.3 Software (Molecular Devices, Sunnyvale, USA).

\section{Results and Discussion}

All the twenty four synthesized compounds $\mathbf{3} \mathbf{a} a-\mathbf{h b}$ were screened for their antimicrobial activities against four pathogenic bacterias, $E$. coli $(\mathrm{Ec})$, Pseudomonas aeruginosa $(\mathrm{Pa})$, Staphyloccus aerus (Sa) and Klebsiella pneumonia (Kp). The same compounds were also tested against six pathogenic fungi Candida albicans (Ca), Cryptococcus neoformans (Cn), Sporothrix schenckii (Ss), Trichophyton mentagrophytes (Tm), Aspergillus fumigatus (Af) and Candida parapsilosis (Cp). Biological studies of the compounds revealed that substitution at position 7 of 3-substituted coumarins by basic amino-ether side chain showed the MIC values at $>50 \mu \mathrm{g} / \mathrm{mL}$ against bacterial strains but have some significant results against antifungal strains. From the activity results it seems that compounds having diethyl amine, piperidine and pyrrolidine as the basic functionalities showed better activity against the antifungal 
strains. Two compounds $\mathbf{3 a e}$ and $\mathbf{3 b c}$ with the MIC values of $1.56 \mu \mathrm{g} /$ $\mathrm{mL}$ are better than Fluconazole against Trichophyton mentagrophytes.

\section{Conclusions}

A novel class of 3,7-disubstituted 2H-1-benzopyran-2-one were synthesized and evaluated for their anti-fungal and anti-bacterial activities for the first time. The compounds were found inactive against different strains of bacteria but some of these compounds 3ac, 3ae, 3bb, 3bc showed significant activity against selective fungal strains. Compound 3ae and 3bc with the MIC values of $1.56 \mu \mathrm{g} / \mathrm{mL}$ are better than the standard drug Fluconazole against Trichophyton mentagrophytes.

\section{Acknowledgements}

Shailesh Kumar is thankful to the University Grants Commission (UGC), New Delhi for the financial support. SK is thankful to the Sophisticated Analytical Instrument Facility (SAIF), CSIR-CDRI, Lucknow, for providing spectroscopic and analytical data. SK is also thankful to Dr. PK Shukla, Fermentation Technology Division, CSIR-CDRI, Lucknow, for the biological screening of the compounds. SK is also thankful to Director, CSIR-CDRI for support and facilities, and Vice Chancellor, Babasaheb Bhimrao Ambedkar University (BBAU), Lucknow, for support and facilities. The authors confirm that there is no conflict of interest with the commercial identities used inside the manuscript.

\section{References}

1. Murray RD (1989) Coumarins. Natural Product Reports 6: 591-624.

2. Estévez-Braun A, González AG (1997) Coumarins. Nat Prod Rep 14: 465-475.

3. Pratap R, Ram VJ (2014) Natural and synthetic chromenes, fused chromenes, and versatility of dihydrobenzo[h]chromenes in organic synthesis. Chem Rev 114: 10476-10526.

4. Borges F, Roleira F, Milhazes N, Santana L, Uriarte E (2005) Simple coumarins and analogues in medicinal chemistry: occurrence, synthesis and biological activity. Curr Med Chem 12: 887-916.

5. Lacy A, O'Kennedy R (2004) Studies on coumarins and coumarin-related compounds to determine their therapeutic role in the treatment of cancer. Curr Pharm Des 10: 3797-3811.

6. Kostova I (2005) Synthetic and natural coumarins as cytotoxic agents. Curr Med Chem Anticancer Agents 5: 29-46.

7. Kontogiorgis CA, Hadjipavlou-Litina DJ (2005) Synthesis and antiinflammatory activity of coumarin derivatives. J Med Chem 48: 6400-6408.

8. Fylaktakidou KC, Hadjipavlou-Litina DJ, Litinas KE, Nicolaides DN (2004) Natural and synthetic coumarin derivatives with anti-inflammatory/ antioxidant activities. Curr Pharm Des 10: 3813-3833.

9. Kawate T, Iwase N, Shimizu M, Stanley SA, Wellington S, et al. (2013) Synthesis and structure-activity relationships of phenyl-substituted coumarins with anti-tubercular activity that target FadD32. Bioorg Med Chem Lett 23: 6052-6059.

10. Ramesh B, Pugalendi KV (2006) Antihyperglycemic effect of umbelliferone in streptozotocin-diabetic rats. J Med Food 9: 562-566.

11. Dwivedi AP, Kumar S, Varshney V, Singh AB, Srivastava AK, et al. (2008) Synthesis and antihyperglycemic activity of novel $\mathrm{N}$-acyl-2-arylethylamines and $\mathrm{N}$-acyl-3-coumarylamines. Bioorg Med Chem Lett 18: 2301-2305.

12. Matos MJ, Viña D, Janeiro P, Borges F, Santana L, et al. (2010) New halogenated 3-phenylcoumarins as potent and selective MAO-B inhibitors. Bioorg Med Chem Lett 20: 5157-5160.

13. Matos MJ, Vazquez-Rodriguez S, Uriarte E, Santana L, Viña D (2011) MAO inhibitory activity modulation: 3-Phenylcoumarins versus 3-benzoylcoumarins. Bioorg Med Chem Lett 21: 4224-4227.

14. Ojala T, Remes S, Haansuu P, Vuorela H, Hiltunen R, et al. (2000) Antimicrobial activity of some coumarin containing herbal plants growing in Finland. J Ethnopharmacol 73: 299-305.
15. Kawase M, Varu B, Shah A, Motohashi N, Tani S, et al. (2001) Antimicrobial activity of new coumarin derivatives. Arzneimittelforschung 51: 67-71.

16. Curir P, Galeotti F, Dolci M, Barile E, Lanzotti V (2007) Pavietin, a coumarin from Aesculus pavia with antifungal activity. J Nat Prod 70: 1668-1671.

17. Montagner C, de Souza SM, Groposoa C, Delle Monache F, Smânia EF, et al (2008) Antifungal activity of coumarins. Z Naturforsch C 63: 21-28.

18. Tegtmeier M, Legrum W (1998) 7-Aminocoumarins are substrates of cytochrome P450-isozymes. Arch Pharm (Weinheim) 331: 143-148.

19. Navarro-García VM, Rojas G, Avilés M, Fuentes M, Zepeda G (2011) In vitro antifungal activity of coumarin extracted from Loeselia mexicana Brand. Mycoses 54: e569-571.

20. Dakanali M, Roussakism E, Kay A, Katerinopoulos HE (2005) Tetrahedron Lett 46: 4193.

21. Hadjipavlou-Litina D, Kontogiorgis C, Pontiki E, Dakanali M, Akoumianaki A et al. (2007) Anti-inflammatory and antioxidant activity of coumarins designed as potential fluorescent zinc sensors. J Enzyme Inhib Med Chem 22: 287-292.

22. Nofal ZM, El-Zahar MI, Abd El-Karim SS (2000) Novel Coumarin Derivatives with Expected Biological Activity. Molecules 5: 99-113.

23. Montagner C, de Souza SM, Groposoa C, Delle Monache F, Smânia EF, et al. (2008) Antifungal activity of coumarins. Z Naturforsch C 63: 21-28.

24. Batra N, Batra S, Prateek A, Prakash BN (2012) Prevalence of preventable medication-related hospitalizations in Australia: an opportunity to reduce harm. Int J Pharm 24: 239-249.

25. Negi AS, Chaturvedi D, Gupta A, Ray S, Dwivedy A, et al. (2005) Amide derivatives of 9,11-seco-estra-1,3,5(10)-trien-11-oic acid as modified orally active estrogen agonists with moderate antagonistic activity. Bioorg Med Chem Lett 15: 99-102.

26. Chaturvedi D, Ray S, Srivastava AK, Chander R (2008) Omega-(2-Naphthyloxy) amino alkanes as a novel class of anti-hyperglycemic and lipid lowering agents. Bioorg Med Chem 16: 2489-2498.

27. Chaturvedi D, Chaturvedi AK, Mishra N, Mishra V (2012) An efficient and nove approach for the synthesis of substituted $\mathrm{N}$-aryl lactams. Org Biomol Chem 10 9148-9151.

28. Chaturvedi D, Dwivedi PK, Chaturvedi AK, Mishra N, Siddiqui $\mathrm{HH}$, et al. (2015) Semisynthetic hybrids of boswellic acids: a novel class of potential antiinflammatory and anti-arthritic agents. Med Chem Res 24: 2799-2812.

29. Kumar S, Deshpande S, Chandra V, Kitchlu S, Dwivedi A, et al. (2009) Synthesis and biological evaluation of 2,3,4-triarylbenzopyran derivatives as SERM and therapeutic agent for breast cancer. Bioorg Med Chem 17: 68326840 .

30. Kumar S, Shakya N, Gupta S, Sarkar J, Sahu DP (2009) Synthesis and biological evaluation of novel 4-(hetero) aryl-2-piperazino quinazolines as antileishmanial and anti-proliferative agents. Bioorg Med Chem Lett 19: 2542-2545.

31. Kumar S, Dwivedi AP, Kashyap VK, Saxena AK, Dwivedi AK, et al. (2013) Synthesis and biological evaluation of trans 6-methoxy-1,1-dimethyl-2-phenyl3-aryl-2,3-dihydro-1H-inden-4-yloxyalkylamine derivatives against drug susceptible, non-replicating M. tuberculosis H37Rv and clinical multidrug resistant strains. Bioorg Med Chem Lett 23: 2404-2407. 